\title{
Hierarchical Matrix Approximation for the Uncertainty Quantification of Potentials on Random Domains ${ }^{\text {th }}$
}

\author{
Jürgen Dölz ${ }^{\mathrm{a}}$, Helmut Harbrecht ${ }^{\mathrm{a}}$ \\ ${ }^{a}$ Departement Mathematik und Informatik, Universität Basel, Spiegelgasse 1, 4051 Basel, Switzerland
}

\begin{abstract}
Computing statistical quantities of interest of the solution of PDE on random domains is an important and challenging task in engineering. We consider the computation of these quantities by the perturbation approach. Especially, we discuss how third order accurate expansions of the mean and the correlation can numerically be computed. These expansions become even fourth order accurate for certain types of boundary variations. The correction terms are given by the solution of correlation equations in the tensor product domain, which can efficiently be computed by means of $\mathcal{H}$-matrices. They have recently been shown to be an efficient tool to solve correlation equations with rough data correlations, that is, with low Sobolev smoothness or small correlation length, in almost linear time. Numerical experiments in three dimensions for higher order ansatz spaces show the feasibility of the proposed algorithm. The application to a non-smooth domain is also included.
\end{abstract}

Keywords: uncertainty quantification, perturbation approach, random domains, hierarchical matrices, boundary element method

2010 MSC: 60H35, 65C30, 65N12, 65N15, 65N38

\section{Introduction}

The numerical solution of strongly elliptic linear partial differential equations (PDEs) is an important task in science and engineering. It is nowadays well understood and can be accomplished up to high accuracy, provided that the input data are known exactly. Motivated by tolerances in manufacturing processes and measurement errors, the computation of statistical output functionals of the solution of PDEs on objects with uncertain shapes has recently gained a lot of interest. The domain mapping approach, see e.g. [1, 2, 3, is well suited for modelling large variations in the domain, but usually leads to high-dimensional and costly integration problems, which suffer from the curse of dimensionality. The perturbation approach, see e.g. [4, [5, 6, 7], is motivated by small disturbances in manufacturing processes and models uncertain small deformations under the following view point. Notice that both approaches have recently been combined in [8].

Given a reference domain $D_{0}$, the random domains $D_{\varepsilon}(\omega)$ are defined by the perturbation of the reference domain's boundary in some $\varepsilon$ tube, leading to the model problem

$$
\begin{aligned}
\Delta u_{\varepsilon}(\omega)=0 & \text { in } D_{\varepsilon}(\omega), \\
u_{\varepsilon}(\omega)=g & \text { on } \partial D_{\varepsilon}(\omega) .
\end{aligned}
$$

Using shape calculus, cf. 9, 10, and under some smoothness assumptions, the non-linear dependence of the solution $u_{\varepsilon}(\omega)$ on $D_{\varepsilon}(\omega)$ can, in a suitable compact subdomain $K$, be expanded in a

\footnotetext{
ॠ This research has been supported by the Swiss National Science Foundation (SNSF) through the project "Hmatrix based first and second moment analysis".

Email addresses: Juergen.Doelz@unibas.ch (Jürgen Dölz), Helmut.Harbrecht@unibas.ch (Helmut Harbrecht)
} 
Taylor expansion in $\varepsilon$, i.e.,

$$
u_{\varepsilon}(\omega)=u_{0}+\varepsilon \delta u(\omega)+\frac{\varepsilon^{2}}{2} \delta^{2} u(\omega)+\mathcal{O}\left(\varepsilon^{3}\right) \quad \text { in } K .
$$

The zero order term $u_{0}$ can directly be computed by solving the deterministic PDE

$$
\begin{aligned}
\Delta u_{0}=0 & \text { in } D_{0}, \\
u_{0}=g & \text { on } \partial D_{0},
\end{aligned}
$$

on the unperturbed domain. The first and second order correction terms can be computed by solving the very same equation as for the zero order term, but with different boundary conditions.

Based on the Taylor expansion (1), statistical quantities of the solution like the mean

$$
\mathbb{E}\left[u_{\varepsilon}\right]=u_{0}+\varepsilon \mathbb{E}[\delta u]+\frac{\varepsilon^{2}}{2} \mathbb{E}\left[\delta^{2} u\right]+\mathcal{O}\left(\varepsilon^{3}\right) \quad \text { in } K,
$$

the covariance

$$
\operatorname{Cov}\left[u_{\varepsilon}\right]=\varepsilon^{2} \operatorname{Cor}[\delta u]+\mathcal{O}\left(\varepsilon^{3}\right) \quad \text { in } K,
$$

and the correlation

$\operatorname{Cor}\left[u_{\varepsilon}\right]=u_{0} \otimes u_{0}+\varepsilon\left(u_{0} \otimes \mathbb{E}[\delta u]+\mathbb{E}[\delta u] \otimes u_{0}\right)+\varepsilon^{2}\left(\operatorname{Cor}[\delta u]+\frac{u_{0}}{2} \otimes \mathbb{E}\left[\delta^{2} u\right]+\mathbb{E}\left[\delta^{2} u\right] \otimes \frac{u_{0}}{2}\right)+\mathcal{O}\left(\varepsilon^{3}\right)$ in $K$

can be expanded into asymptotic expansions in $\varepsilon$. It has already been shown in [7] that the second order correction term $\operatorname{Cor}[\delta u]$ is the solution to a correlation equation in the higher-dimensional product domain $D_{0} \times D_{0}$. While the first and second order correction terms $\mathbb{E}[\delta u]$ and $\mathbb{E}\left[\delta^{2} u\right]$ of the mean are given as the solution of PDEs on $D_{0}$, the computation of the boundary values for $\mathbb{E}\left[\delta^{2} u\right]$ has not been investigated yet. We show that they can likewise be obtained by the solution of a correlation equation, but in the product domain $\partial D_{0} \times \partial D_{0}$. As this equation lives solely on the domain boundary, the boundary element method is an obvious discretization method for its solution. Hence, we will use it as a discretization scheme for all occurring equations, omitting the meshing of $D_{0}$. We provide the full convergence analysis for the proposed discretization scheme and slightly relax the assumptions from [7] on the boundary perturbations on our way. Additionally, we remark that the asymptotics can even be up to fourth order accurate, if the law of the prescribed boundary variations behaves in a specific way.

We therefore have to solve two correlation equations in the tensor product domain in order to compute third order accurate approximations in the perturbation amplitude $\varepsilon$. As a naive discretization of these higher dimensional problems, also referred to as the full tensor approach, is prohibitively expensive, the solution of such correlation equations has been the topic of several articles, cf. [5, 7, 11, 12, 13, 14, 15, 16, for example. Except for [5, 12, where a low-rank approximation of the underlying correlation is employed, all of the mentioned approaches rely in some sense on a sparse tensor approximation. Both, low-rank approximations and sparse tensor discretizations, are best suitable if the prescribed correlation is sufficiently smooth, compare [17, 18, for the behaviour of low-rank approximations in dependence of the smoothness, and are known to struggle for "rough" correlations. This means that the prescribed correlation exhibits only minor smoothness assumptions or has a high concentration of measure. While rough correlations do not necessarily have an influence on the convergence rates ([19] discusses an specific example where the rate is reduced), they may have a huge influence on the constants involved in the complexity estimates.

Recently, the hierarchical matrix approach (in short $\mathcal{H}$-matrix approach) to correlation equations, cf. [19, 20, 21], has been shown to be a promising approach to cope with rough correlations. In the context of correlation equations, $\mathcal{H}$-matrices provide an alternative compression scheme to represent the full tensor approach and allow the solution of correlation equations in almost linear time. Being introduced in [22, 23, $\mathcal{H}$-matrices are feasible for the data-sparse representation of (block-) matrices which can be approximated block-wise with low-rank. They have originally been 


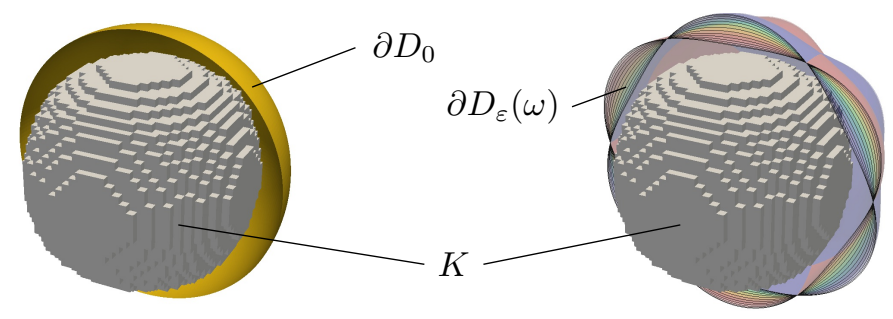

Figure 1: The boundary of the reference domain $\partial D_{0}$, a family of perturbed domains $\partial D_{\varepsilon}(\omega)$, and the compactum K.

employed for the efficient treatment of boundary integral equations, as they arise in the boundary element method. Nonetheless, they also provide an arithmetic which can be employed for the solution of matrix equations, as they occur from the discretization of correlation equations.

The rest of this article is organized as follows. In Section 2, we derive expansions of the mean, the covariance, and the correlation with respect to the perturbation's amplitude via shape calculus. Section 3 is concerned with the necessary boundary integral equations to allow for a natural treatment of the random perturbations of the boundary. In Section 4, we introduce the corresponding Galerkin discretization, whereas Section 5 is concerned with its error estimation. Section 6 is concerned with the efficient treatment of the derived equations with $\mathcal{H}$-matrices. We demonstrate the feasibility of the proposed approach by numerical experiments in three spatial dimensions in Section 7. Finally, in Section 8, we draw our conclusions.

\section{Random Domains}

\subsection{Basic Definitions}

For the following considerations, let $D_{0} \subset \mathbb{R}^{n}$ be a reference domain with, in order to ensure $C^{3,1}$-continuity of its outer normal, a $C^{4,1}$-boundary. On a separable, complete probability space $(\Omega, \Sigma, \mathbb{P})$, consider a random vector field $\mathbf{V} \in L_{\mathbb{P}}^{2}\left(\Omega ; C^{3,1}\left(\partial D_{0}, \mathbb{R}^{n}\right)\right)$ with $\|\mathbf{V}(\omega, \cdot)\|_{C^{3,1}\left(\partial D_{0} ; \mathbb{R}^{n}\right)} \lesssim 1$ uniformly for all $\omega \in \Omega$, which perturbs the boundary of the reference domain $\partial D_{0}$ in accordance with $\partial D_{\varepsilon}(\omega):=\partial D_{0}+\varepsilon \mathbf{V}\left(\omega, \partial D_{0}\right)$ for some given $\varepsilon>0$. A random domain $D_{\varepsilon}(\omega)$ is then given by the interior of the perturbed boundary $\partial D_{\varepsilon}(\omega)$. For later considerations, we also introduce a compact set $K$, which is contained in all possible boundary variations and, in particular, in the reference domain, i.e.,

$$
K \Subset D_{0} \cap D_{\varepsilon}^{\cap \Omega}, \quad D_{\varepsilon}^{\cap \Omega}:=\bigcap_{\omega \in \Omega} D_{\varepsilon}(\omega) .
$$

The complete setting of the introduced sets is illustrated in Figure 1 .

Note that, in contrast to the domain mapping approach, which requires a vector field on the whole reference domain, the perturbation approach only requires a vector field on the boundary. A correspondence between the two approaches is given by the fact that every vector field on the boundary $\partial D_{0}$ can smoothly be extended into the interior of $D_{0}$ in such a way that it vanishes on the compactum $K$.

For all further considerations, let $g \in H^{3}\left(D_{0} \cup D_{\varepsilon}^{\cup \Omega}\right)$, where $D_{\varepsilon}^{\cup \Omega}$ denotes the hold-all domain

$$
D_{\varepsilon}^{\cup \Omega}:=\bigcup_{\omega \in \Omega} D_{\varepsilon}(\omega) .
$$

Then, it follows $u_{0} \in H^{3}\left(D_{0}\right)$ for the unique solution $u_{0}$ of the unperturbed problem

$$
\begin{aligned}
\Delta u_{0}=0 & \text { in } D_{0}, \\
u_{0}=g & \text { on } \partial D_{0},
\end{aligned}
$$

see [24, Theorem 20.4] for example. Here and in the following, the Dirichlet and Neumann data have to be understood in the trace sense. 


\subsection{Shape Calculus for Parametrized Domains}

We look at the randomly perturbed boundary value problem

$$
\begin{aligned}
\Delta u_{\varepsilon}(\omega)=0 & \text { in } D_{\varepsilon}(\omega), \\
u_{\varepsilon}(\omega)=g & \text { on } \partial D_{\varepsilon}(\omega),
\end{aligned}
$$

which is posed on the random domain $D_{\varepsilon}(\omega)$. To deal with the non-linear dependence of $u_{\varepsilon}(\omega)$ on $D_{\varepsilon}(\omega)$, we may exploit that the dependence is Fréchet-differentiable, see, e.g., [25. More precisely, given the reference domain $D_{0}$ and the vector field $\mathbf{V} \in L_{\mathbb{P}}^{2}\left(\Omega ; C^{3,1}\left(\partial D_{0} ; \mathbb{R}^{n}\right)\right)$ with $\|\mathbf{V}(\omega, \cdot)\|_{C^{3,1}\left(\partial D_{0} ; \mathbb{R}^{n}\right)} \lesssim 1$ uniformly for all $\omega \in \Omega$, one can expand $u_{\varepsilon}$ into a shape Taylor expansion

$$
\begin{aligned}
& u_{\varepsilon}(\omega, \mathbf{x})=u_{0}(\mathbf{x})+\varepsilon \delta u[\mathbf{V}(\omega)](\mathbf{x})+\frac{\varepsilon^{2}}{2} \delta^{2} u[\mathbf{V}(\omega), \mathbf{V}(\omega)](\mathbf{x})+R_{2}(\varepsilon \mathbf{V}(\omega))(\mathbf{x}), \\
& \mathbf{x} \in K \Subset D_{0} \cap D_{\varepsilon}(\omega),
\end{aligned}
$$

which holds for all $0<\varepsilon \leq \varepsilon_{0}$ for some $\varepsilon_{0}>0$ small enough. Here, the first order local shape derivative $\delta u[\mathbf{V}(\omega)] \in H^{2}\left(D_{0}\right)$ is given by

$$
\begin{aligned}
\Delta \delta u[\mathbf{V}(\omega)] & =0 & & \text { in } D_{0}, \\
\delta u[\mathbf{V}(\omega)] & =\langle\mathbf{V}(\omega), \mathbf{n}\rangle \frac{\partial\left(g-u_{0}\right)}{\partial \mathbf{n}} & & \text { on } \partial D_{0},
\end{aligned}
$$

cf. [9, Chapter 10] and [10, Chapter 3.1]. Given a second vector field $\mathbf{V}^{\prime} \in L_{\mathbb{P}}^{2}\left(\Omega ; C^{3,1}\left(D_{0} ; \mathbb{R}^{n}\right)\right)$ for which it holds $\left\|\mathbf{V}^{\prime}(\omega, \cdot)\right\|_{C^{3,1}\left(D_{0} ; \mathbb{R}^{n}\right)} \lesssim 1$ uniformly for all $\omega \in \Omega$, the second order local shape derivative $\delta^{2} u\left[\mathbf{V}(\omega), \mathbf{V}^{\prime}(\omega)\right] \in H^{1}\left(D_{0}\right)$ is given, cf. [25, Theorem 1], by

$$
\begin{aligned}
\Delta \delta^{2} u\left[\mathbf{V}(\omega), \mathbf{V}^{\prime}(\omega)\right] & =0 & \text { in } D_{0}, \\
\delta^{2} u\left[\mathbf{V}(\omega), \mathbf{V}^{\prime}(\omega)\right]=\frac{\partial^{2}\left(g-u_{0}\right)}{\partial \mathbf{n}^{2}} & \langle\mathbf{V}(\omega), \mathbf{n}\rangle\left\langle\mathbf{V}^{\prime}(\omega), \mathbf{n}\right\rangle & \\
& -\langle\mathbf{V}(\omega), \mathbf{n}\rangle \frac{\partial \delta u\left[\mathbf{V}^{\prime}(\omega)\right]}{\partial \mathbf{n}} & \\
& -\left\langle\mathbf{V}^{\prime}(\omega), \mathbf{n}\right\rangle \frac{\partial \delta u[\mathbf{V}(\omega)]}{\partial \mathbf{n}} & \text { on } \partial D_{0} .
\end{aligned}
$$

Of course, according to (4), we only need the second order local shape derivative in case of identical fields, i.e., $\mathbf{V}=\mathbf{V}^{\prime}$ and it only remains to explain how to compute $\partial^{2}\left(g-u_{0}\right) / \partial \mathbf{n}^{2}$ on $\partial D_{0}$. On a $C^{2}$-boundary and for $\varphi \in H^{3}(D)$, we know that, cf. [10, Proposition 2.68],

$$
\Delta \varphi=\Delta_{\Gamma} \varphi+2 \mathcal{H} \frac{\partial \varphi}{\partial \mathbf{n}}+\frac{\partial^{2} \varphi}{\partial \mathbf{n}^{2}} \quad \text { on } \partial D_{0},
$$

where $\Delta_{\Gamma}$ denotes the Laplace-Beltrami operator and $\mathcal{H}$ denotes the mean curvature of $\partial D_{0}$ given by $2 \mathcal{H}=-\nabla \cdot \mathbf{n}$. Since $u_{0}=g$ on $\partial D_{0}$, see $(2)$, we have $\Delta_{\Gamma}\left(g-u_{0}\right)=0$, and obtain

$$
\frac{\partial^{2}\left(g-u_{0}\right)}{\partial \mathbf{n}^{2}}=\Delta g-2 \mathcal{H} \frac{\partial\left(g-u_{0}\right)}{\partial \mathbf{n}} \text { on } \partial D_{0}
$$

by the use of (7).

By definition of the Fréchet derivative, the remainder $R_{2}$ in $(4)$ is uniformly in $\varepsilon \mathbf{V}(\omega)$ negligible with respect to $\|\varepsilon \mathbf{V}(\omega)\|_{C^{3,1}\left(\partial D_{0}\right)}^{2}$, i.e., $R_{2}(\varepsilon \mathbf{V}(\omega))=o\left(\|\varepsilon \mathbf{V}(\omega)\|_{C^{3,1}\left(\partial D_{0}\right)}^{2}\right)$. However, one can show that the second order correction term satisfies a Lipschitz-condition, see [26]. Together with the assumption that $\|\mathbf{V}(\omega, \cdot)\|_{C^{3,1}\left(\partial D_{0} ; \mathbb{R}^{n}\right)} \lesssim 1$ uniformly for all $\omega \in \Omega$, this allows to show that

$$
R_{2}(\varepsilon \mathbf{V}(\omega))=\mathcal{O}\left(\varepsilon^{3}\right)
$$

where the constant is independent of $\omega$. 


\subsection{Statistical Moments on Random Domains}

For the following considerations, we can assume without loss of generality that the boundary perturbations are centered, i.e.,

$$
\mathbb{E}[\mathbf{V}]=\mathbf{0}, \quad \text { and thus formally } \mathbb{E}\left[D_{\varepsilon}\right]=D_{0},
$$

which is not a restriction, since one can easily recenter the random field by considering $\mathbf{V}^{\prime}=$ $\mathbf{V}-\mathbb{E}[\mathbf{V}]$. This especially means that the mean of the first order local shape derivative (5) vanishes, which implies, using $(9)$ and taking the mean of (4),

$$
\mathbb{E}\left[u_{\varepsilon}\right](\mathbf{x})=u_{0}(\mathbf{x})+\frac{\varepsilon^{2}}{2} \mathbb{E}\left[\delta^{2} u\right](\mathbf{x})+\mathcal{O}\left(\varepsilon^{3}\right), \quad \mathbf{x} \in K \Subset D_{\varepsilon}^{\cap \Omega} .
$$

By using (9), tensorizing (4), and integrating over $\Omega$, we further obtain

$$
\begin{aligned}
\operatorname{Cor}\left[u_{\varepsilon}\right](\mathbf{x}, \mathbf{y})=u_{0}(\mathbf{x}) & u_{0}(\mathbf{y})+\varepsilon^{2} \operatorname{Cor}[\delta u](\mathbf{x}, \mathbf{y}) \\
+ & \frac{\varepsilon^{2}}{2}\left(u_{0}(\mathbf{x}) \mathbb{E}\left[\delta^{2} u\right](\mathbf{y})+\mathbb{E}\left[\delta^{2} u\right](\mathbf{x}) u_{0}(\mathbf{y})\right)+\mathcal{O}\left(\varepsilon^{3}\right), \quad \mathbf{x}, \mathbf{y} \in K \Subset D_{\varepsilon}^{\cap \Omega} .
\end{aligned}
$$

Finally, using (9), subtracting $(10)$ from both sides in (4), and tensorizing and integrating over $\Omega$ yields

$$
\operatorname{Cov}\left[u_{\varepsilon}\right](\mathbf{x}, \mathbf{y})=\varepsilon^{2} \operatorname{Cor}[\delta u](\mathbf{x}, \mathbf{y})+\mathcal{O}\left(\varepsilon^{3}\right), \quad \mathbf{x}, \mathbf{y} \in K \Subset D_{\varepsilon}^{\cap \Omega} .
$$

In order to compute the quantities $\mathbb{E}\left[u_{\varepsilon}\right], \operatorname{Cor}\left[u_{\varepsilon}\right]$, and $\operatorname{Cov}\left[u_{\varepsilon}\right]$, appearing in (10), (11), and $\left[12\right.$, we have to solve for $\mathbb{E}\left[\delta^{2} u\right]$ and $\operatorname{Cor}[\delta u]$. To this end, combing (6) and (8), taking the mean, and interchanging integration and differentiation yields, see also [7,

$$
\begin{array}{rlrl}
\Delta \mathbb{E}\left[\delta^{2} u[\mathbf{V}, \mathbf{V}]\right] & =0 & \text { in } D_{0}, \\
\mathbb{E}\left[\delta^{2} u[\mathbf{V}, \mathbf{V}]\right]=\left.\left(\Delta g-2 \mathcal{H} \frac{\partial\left(g-u_{0}\right)}{\partial \mathbf{n}}\right) \operatorname{Cor}[\langle\mathbf{V}, \mathbf{n}\rangle]\right|_{\mathbf{x}=\mathbf{y}}-2 \mathbb{E}\left[\langle\mathbf{V}, \mathbf{n}\rangle \frac{\partial \delta u[\mathbf{V}]}{\partial \mathbf{n}}\right] & \text { on } \partial D_{0} .
\end{array}
$$

Next, tensorizing (5), integrating over $\Omega$, and interchanging integration and differentiation yields

$$
\begin{aligned}
(\Delta \otimes \Delta) \operatorname{Cor}[\delta u] & =0 & & \text { in } D_{0} \times D_{0}, \\
(\Delta \otimes \operatorname{Id}) \operatorname{Cor}[\delta u] & =0 & & \text { on } D_{0} \times \partial D_{0}, \\
(\operatorname{Id} \otimes \Delta) \operatorname{Cor}[\delta u] & =0 & & \text { on } \partial D_{0} \times D_{0}, \\
\operatorname{Cor}[\delta u] & =\operatorname{Cor}[\langle\mathbf{V}, \mathbf{n}\rangle]\left(\frac{\partial\left(g-u_{0}\right)}{\partial \mathbf{n}} \otimes \frac{\partial\left(g-u_{0}\right)}{\partial \mathbf{n}}\right) & & \text { on } \partial D_{0} \times \partial D_{0}
\end{aligned}
$$

The asymptotic expansions for $\mathbb{E}\left[u_{\varepsilon}\right], \operatorname{Cor}\left[u_{\varepsilon}\right]$, and $\operatorname{Cov}\left[u_{\varepsilon}\right]$ can, under certain circumstances, be improved to fourth order accuracy with help of the following lemma, which is inspired by [6. Lemma 2.3] and [27, Chapter XI].

Lemma 2.1. Assume that the boundary perturbations in normal direction are given by an expansion

$$
\langle\mathbf{V}(\omega, \mathbf{x}), \mathbf{n}\rangle=\sum_{i=1}^{M} \kappa_{i}(\mathbf{x}) X_{i}(\omega),
$$

where $X_{i}, i=1, \ldots, M$, are independent and identically distributed random variables. Then, it holds

$$
\begin{aligned}
\delta u[\mathbf{V}(\omega)] & =\sum_{i=1}^{M} \delta u\left[\kappa_{i} \cdot \mathbf{n}\right] X_{i}(\omega) \\
\delta^{2} u[\mathbf{V}(\omega), \mathbf{V}(\omega)] & =\sum_{i, j=1}^{M} \delta^{2} u\left[\kappa_{i} \cdot \mathbf{n}, \kappa_{j} \cdot \mathbf{n}\right] X_{i}(\omega) X_{j}(\omega),
\end{aligned}
$$


and

$$
\delta^{3} u[\mathbf{V}(\omega), \mathbf{V}(\omega), \mathbf{V}(\omega)]=\sum_{i, j, k=1}^{M} \delta^{3} u\left[\kappa_{i} \cdot \mathbf{n}, \kappa_{j} \cdot \mathbf{n}, \kappa_{k} \cdot \mathbf{n}\right] X_{i}(\omega) X_{j}(\omega) X_{k}(\omega),
$$

provided that the third order local shape derivative $\delta^{3} u$, as usual given as the local shape derivative of the second order local shape derivative, exists.

Proof. The first two expressions were already provided in [6, Lemma 2.3], whereas the third one is analogously derived by exploiting the trilinearity of $\delta^{3} u$.

Obviously, due to the independence of the random variables $\left(X_{i}\right)_{i=1}^{M}$, it holds

$$
\mathbb{E}\left[\delta^{3} u\right]=\sum_{i=1}^{M} \delta^{3} u\left[\kappa_{i} \cdot \mathbf{n}, \kappa_{i} \cdot \mathbf{n}, \kappa_{i} \cdot \mathbf{n}\right] \mathbb{E}\left[X_{i}^{3}\right]=0,
$$

if the probability distribution of the $X_{i}$ is symmetric around zero. The expansion of the mean $\mathbb{E}\left[u_{\varepsilon}\right][10)$ is thus fourth order accurate if we assume that the third order local shape derivative is Lipschitz-continuous. Similarly, it holds under the same assumptions

$$
\operatorname{Cor}\left[\delta^{2} u, \delta u\right]=\sum_{i=1}^{M} \delta^{2} u\left[\kappa_{i} \cdot \mathbf{n}, \kappa_{i} \cdot \mathbf{n}\right] \delta u_{0}\left[\kappa_{i} \cdot \mathbf{n}\right] \mathbb{E}\left[X_{i}^{3}\right]=0 .
$$

Hence, the expansion for Cor $\left[u_{\varepsilon}\right]$ (11) becomes fourth order accurate, and, likewise, the expansion for $\operatorname{Cov}\left[u_{\varepsilon}\right](12)$ also becomes fourth order accurate.

\section{Boundary Integral Equations}

We shall use boundary integral equations to compute the asymptotic expansions $(10$, , 11), and 12 for the statistics of the random solution. To this end, observe that the boundary conditions for the PDEs of $\mathbb{E}\left[\delta^{2} u\right]$ and Cor $[\delta u],(13)$ and (14), depend on the Neumann data $t^{\left(u_{0}\right)}=\partial u_{0} / \partial \mathbf{n}$ of the solution $u_{0}$ of the unperturbed problem (2). Having the Dirichlet data $g$ of $u_{0}$ at hand, the corresponding Neumann data are given by the Dirichlet-to-Neumann map,

$$
\mathcal{S}: H^{1 / 2}\left(\partial D_{0}\right) \rightarrow H^{-1 / 2}\left(\partial D_{0}\right), \quad \mathcal{S}:=\mathcal{V}^{-1}\left(\frac{1}{2}+\mathcal{K}\right), \quad g \mapsto t^{\left(u_{0}\right)},
$$

where the single layer operator is given by

$$
\mathcal{V}: H^{-1 / 2}\left(\partial D_{0}\right) \rightarrow H^{1 / 2}\left(\partial D_{0}\right), \quad(\mathcal{V} \rho)(\mathbf{x})=\int_{\partial D_{0}} \frac{\rho(\mathbf{y})}{4 \pi\|\mathbf{x}-\mathbf{y}\|} \mathrm{d} \sigma_{\mathbf{y}}, \quad \mathbf{x} \in \partial D_{0},
$$

and the double layer operator is given by

$$
\mathcal{K}: H^{1 / 2}\left(\partial D_{0}\right) \rightarrow H^{1 / 2}\left(\partial D_{0}\right), \quad(\mathcal{K} \rho)(\mathbf{x})=\int_{\partial D_{0}} \frac{\left\langle\mathbf{x}-\mathbf{y}, \mathbf{n}_{\mathbf{y}}\right\rangle \rho(\mathbf{y})}{4 \pi\|\mathbf{x}-\mathbf{y}\|^{3}} \mathrm{~d} \sigma_{\mathbf{y}}, \quad \mathbf{x} \in \partial D_{0},
$$

cf. e.g. [28].

To obtain the second term of the Dirichlet data of $\mathbb{E}\left[\delta^{2} u\right]$ in $[13$, we employ that it can be equivalently rewritten as the diagonal of a correlation, call it $A$,

$$
\mathbb{E}\left[\langle\mathbf{V}, \mathbf{n}\rangle \frac{\partial \delta u[\mathbf{V}]}{\partial \mathbf{n}}\right]=\left.\mathbb{E}\left[\left\langle\mathbf{V}(\cdot, \mathbf{x}), \mathbf{n}_{\mathbf{x}}\right\rangle \frac{\partial \delta u[\mathbf{V}(\cdot, \mathbf{y})])}{\partial \mathbf{n}_{\mathbf{y}}}\right]\right|_{\mathbf{x}=\mathbf{y}}=:\left.A(\mathbf{x}, \mathbf{y})\right|_{\mathbf{x}=\mathbf{y}} .
$$


The Dirichlet-to-Neumann map and the definition of the first order local shape derivative (5) then yield

$$
\begin{aligned}
A(\mathbf{x}, \mathbf{y}) & :=\mathbb{E}\left[\left\langle\mathbf{V}(\cdot, \mathbf{x}), \mathbf{n}_{\mathbf{x}}\right\rangle \frac{\partial \delta u[\mathbf{V}(\cdot, \mathbf{y})])}{\partial \mathbf{n}_{\mathbf{y}}}\right] \\
& =\mathbb{E}\left[\left\langle\mathbf{V}(\cdot, \mathbf{x}), \mathbf{n}_{\mathbf{x}}\right\rangle \mathcal{S}\left(\left\langle\mathbf{V}(\cdot, \mathbf{y}), \mathbf{n}_{\mathbf{y}}\right\rangle\left(\frac{\partial g}{\partial \mathbf{n}_{\mathbf{y}}}-t^{\left(u_{0}\right)}\right)\right)\right] \\
& =(\operatorname{Id} \otimes \mathcal{S})\left(\mathbb{E}\left[\left\langle\mathbf{V}(\cdot, \mathbf{x}), \mathbf{n}_{\mathbf{x}}\right\rangle\left\langle\mathbf{V}(\cdot, \mathbf{y}), \mathbf{n}_{\mathbf{y}}\right\rangle\right]\left(\mathbb{1} \otimes\left(\frac{\partial g}{\partial \mathbf{n}_{\mathbf{y}}}-t^{\left(u_{0}\right)}\right)\right)\right) \\
& =(\operatorname{Id} \otimes \mathcal{S})\left(\left(\mathbb{1} \otimes\left(\frac{\partial g}{\partial \mathbf{n}_{\mathbf{y}}}-t^{\left(u_{0}\right)}\right)\right) \operatorname{Cor}[\langle\mathbf{V}, \mathbf{n}\rangle]\right) .
\end{aligned}
$$

Given the Dirichlet data

$$
g^{\left(\mathbb{E}\left[\delta^{2} u\right]\right)}=\left.\left(\Delta g-2 \mathcal{H} \frac{\partial\left(g-u_{0}\right)}{\partial \mathbf{n}}\right) \operatorname{Cor}[\langle\mathbf{V}, \mathbf{n}\rangle]\right|_{\mathbf{x}=\mathbf{y}}-\left.2 A\right|_{\mathbf{x}=\mathbf{y}}
$$

and the Neumann data

$$
t^{\left(\mathbb{E}\left[\delta^{2} u\right]\right)}=\mathcal{S} g^{\left(\mathbb{E}\left[\delta^{2} u\right]\right)}
$$

of $\mathbb{E}\left[\delta^{2} u\right]$, the solution $\mathbb{E}\left[\delta^{2} u\right]$ of $[13$ can be represented inside the domain by using the representation formula

$$
\mathbb{E}\left[\delta^{2} u\right](\mathbf{x})=\tilde{\mathcal{V}}\left(t^{\left(\mathbb{E}\left[\delta^{2} u\right]\right)}\right)(\mathbf{x})-\tilde{\mathcal{K}}\left(g^{\left(\mathbb{E}\left[\delta^{2} u\right]\right)}\right)(\mathbf{x}), \quad \mathbf{x} \Subset D_{\cap \Omega} .
$$

Here, the single layer potential is given by

$$
\tilde{\mathcal{V}}: H^{-1 / 2}\left(\partial D_{0}\right) \rightarrow H^{1}\left(D_{0}\right), \quad(\tilde{\mathcal{V}} \rho)(\mathbf{x})=\int_{\partial D_{0}} \frac{\rho(\mathbf{y})}{4 \pi\|\mathbf{x}-\mathbf{y}\|} \mathrm{d} \sigma_{\mathbf{y}}, \quad \mathbf{x} \in D_{\cap \Omega},
$$

and the double layer potential is given by

$$
\tilde{\mathcal{K}}: H^{1 / 2}\left(\partial D_{0}\right) \rightarrow H^{1}\left(D_{0}\right), \quad(\tilde{\mathcal{K}} \rho)(\mathbf{x})=\int_{\partial D_{0}} \frac{\left\langle\mathbf{x}-\mathbf{y}, \mathbf{n}_{\mathbf{y}}\right\rangle \rho(\mathbf{y})}{4 \pi\|\mathbf{x}-\mathbf{y}\|^{3}} \mathrm{~d} \sigma_{\mathbf{y}}, \quad \mathbf{x} \in D_{\cap \Omega}
$$

cf. e.g. 28].

To obtain a boundary reduction of (14), we observe that the solution to 5 can be obtained, see also 28, by solving

and computing

$$
\mathcal{V} \rho^{(\delta u[\mathbf{V}(\omega)])}=\langle\mathbf{V}(\omega), \mathbf{n}\rangle \frac{\partial\left(g-u_{0}\right)}{\partial \mathbf{n}}
$$

$$
\delta u[\mathbf{V}(\omega)](\mathbf{x})=\tilde{\mathcal{V}} \rho^{(\delta u[\mathbf{V}(\omega)])}, \quad \mathbf{x} \in D_{\cap \Omega} .
$$

Tensorizing both equations, integrating over $\Omega$, and applying Fubini's theorem yields that, for given Dirichlet data of $\operatorname{Cor}[\delta u]$,

$$
g^{(\operatorname{Cor}[\delta u])}=\left(\frac{\partial\left(g-u_{0}\right)}{\partial \mathbf{n}} \otimes \frac{\partial\left(g-u_{0}\right)}{\partial \mathbf{n}}\right) \operatorname{Cor}[\langle\mathbf{V}, \mathbf{n}\rangle]
$$

one may solve

$$
(\mathcal{V} \otimes \mathcal{V}) \rho^{(\operatorname{Cor}[\delta u])}=g^{(\operatorname{Cor}[\delta u])}
$$

to compute the second order correction term Cor $[\delta u]$ by the representation formula

$$
\operatorname{Cor}[\delta u](\mathbf{x}, \mathbf{y})=(\tilde{\mathcal{V}} \otimes \tilde{\mathcal{V}})\left(\rho^{(\operatorname{Cor}[\delta u])}\right)(\mathbf{x}, \mathbf{y}), \quad \mathbf{x}, \mathbf{y} \in D_{\cap \Omega},
$$

see also [19] for explicit computations in a similar setting. 


\section{Galerkin Discretization}

For the following discussion of the Galerkin discretizations, let $S_{h}^{d}$ be the $N$-dimensional space of piecewise polynomial ansatz functions of order $d$ on $\partial D_{0}$, generated from a quasi-uniform mesh on $\partial D_{0}$. To improve readability, we choose a basis $\left(\psi_{i}\right)_{i=1}^{N}$ of $S_{h}^{d}$ and introduce the system matrices

$$
\begin{array}{rcrl}
\mathbf{V} & =\left[\left(\mathcal{V} \psi_{j}, \psi_{i}\right)_{L^{2}\left(\partial D_{0}\right)}\right]_{i, j=1}^{N}, & \mathbf{M} & =\left[\left(\psi_{j}, \psi_{i}\right)_{L^{2}\left(\partial D_{0}\right)}\right]_{i, j=1}^{N}, \\
\mathbf{K} & =\left[\left(\mathcal{K} \psi_{j}, \psi_{i}\right)_{L^{2}\left(\partial D_{0}\right)}\right]_{i, j=1}^{N}, & \mathbf{C}_{\operatorname{Cor}[\langle\mathbf{V}, \mathbf{n}\rangle]}=\left[\left(\operatorname{Cor}[\langle\mathbf{V}, \mathbf{n}\rangle], \psi_{i} \otimes \psi_{j}\right)_{L^{2}\left(\partial D_{0} \times \partial D_{0}\right)}\right]_{i, j=1}^{N} .
\end{array}
$$

Moreover, the tensor product between two matrices in the following equations has, as usual, to be understood as the Kronecker product. We will also use the fact that, for matrices $\mathbf{A} \in \mathbb{R}^{k \times n}$, $\mathbf{B} \in \mathbb{R}^{\ell \times m}$ and $\mathbf{X} \in \mathbb{R}^{m \times n}$, there holds the relation

$$
(\mathbf{A} \otimes \mathbf{B}) \operatorname{vec}(\mathbf{X})=\operatorname{vec}\left(\mathbf{B X A}^{\boldsymbol{\top}}\right) .
$$

Here, for $\mathbf{A}=\left[\mathbf{a}_{1}, \ldots, \mathbf{a}_{n}\right] \in \mathbb{R}^{m \times n}$, the operation $\operatorname{vec}(\mathbf{A})$ is defined as

$$
\operatorname{vec}\left(\left[\mathbf{a}_{1}, \ldots, \mathbf{a}_{n}\right]\right):=\left[\begin{array}{c}
\mathbf{a}_{1} \\
\vdots \\
\mathbf{a}_{n}
\end{array}\right] \in \mathbb{R}^{m n} .
$$

\subsection{Dirichlet-to-Neumann Map}

Since the Neumann data $t^{\left(u_{0}\right)}$ of the solution $u_{0}$ of the unperturbed boundary value problem (2) are needed for the computation of both of the correction terms $\mathbb{E}\left[\delta^{2} u\right]$ and Cor $[\delta u]$, we will first consider the discretization of the Dirichlet-to-Neumann map (15). Although the discretization is pretty standard in boundary element methods, cf., e.g., [28, we believe its repetition is useful to establish notation and will help the reader to understand the following more involved steps.

The variational formulation of the Dirichlet-to-Neumann map 15 is given as follows:

$$
\begin{aligned}
& \text { Find } t^{\left(u_{0}\right)} \in H^{-1 / 2}\left(\partial D_{0}\right) \text { such that } \\
& \qquad\left(\mathcal{V} t^{\left(u_{0}\right)}, v\right)_{L^{2}\left(\partial D_{0}\right)}=\left(\left(\frac{1}{2}+\mathcal{K}\right) g, v\right)_{L^{2}\left(\partial D_{0}\right)} \\
& \text { for all } v \in H^{-1 / 2}\left(\partial D_{0}\right) \text {. }
\end{aligned}
$$

Replacing the energy space $H^{-1 / 2}\left(\partial D_{0}\right)$ by the finite dimensional space $S_{h}^{d} \subset H^{-1 / 2}\left(\partial D_{0}\right)$ and replacing $g$ with its $L^{2}$-projection $\Pi_{h} g$ onto $S_{h}^{d}$, we end up with the system of linear equations

$$
\mathbf{V} \mathbf{t}^{\left(u_{0}\right)}=\left(\frac{1}{2} \mathbf{M}+\mathbf{K}\right) \mathbf{M}^{-1} \mathbf{g}
$$

Here, the corresponding vectors are given by

$$
\mathbf{g}=\left[\left(g, \psi_{i}\right)_{L^{2}\left(\partial D_{0}\right)}\right]_{i=1}^{N}, \quad \mathbf{t}^{\left(u_{0}\right)}=\left[t_{i}^{\left(u_{0}\right)}\right]_{i=1}^{N},
$$

where the coefficients $t_{i}^{\left(u_{0}\right)}$ are the coefficients of the basis expansion

$$
t_{h}^{\left(u_{0}\right)}=\sum_{i=1}^{N} t_{i}^{\left(u_{0}\right)} \psi_{i} .
$$




\subsection{Computation of $\operatorname{Cor}[\delta u]$}

For determining $\operatorname{Cor}[\delta u]$ via the representation formula $(23)$, we have to compute $\rho^{(\operatorname{Cor}[\delta u])}$, which is given as the solution of the tensor equation (22). We therefore introduce the Sobolev spaces $H_{\text {mix }}^{s, t}\left(\partial D_{0} \times \partial D_{0}\right), s, t \in \mathbb{R}$, of dominant mixed derivatives on $\partial D_{0} \times \partial D_{0}$ defined by

$$
H_{\text {mix }}^{s, t}\left(\partial D_{0} \times \partial D_{0}\right):=H^{s}\left(\partial D_{0}\right) \otimes H^{t}\left(\partial D_{0}\right) .
$$

The variational formulation of 22 is then given as follows:

Find $\rho^{(\operatorname{Cor}[\delta u])} \in H_{\text {mix }}^{-1 / 2,-1 / 2}\left(\partial D_{0} \times \partial D_{0}\right)$ such that

$$
\left((\mathcal{V} \otimes \mathcal{V}) \rho^{(\operatorname{Cor}[\delta u])}, v\right)_{L^{2}\left(\partial D_{0} \times \partial D_{0}\right)}=\left(g^{(\operatorname{Cor}[\delta u])}, v\right)_{L^{2}\left(\partial D_{0} \times \partial D_{0}\right)}
$$

for all $v \in H_{\text {mix }}^{-1 / 2,-1 / 2}\left(\partial D_{0} \times \partial D_{0}\right)$.

Similar to the discretization of the Dirichlet-to-Neumann map 15 , we introduce $L^{2}$-projections to replace the right-hand side $g^{(\operatorname{Cor}[\delta u])}$ by the approximation

$$
g_{h}^{(\operatorname{Cor}[\delta u])}:=\left(\left(\frac{\partial g}{\partial \mathbf{n}}-t_{h}^{\left(u_{0}\right)}\right) \otimes\left(\frac{\partial g}{\partial \mathbf{n}}-t_{h}^{\left(u_{0}\right)}\right)\right)\left(\left(\Pi_{h} \otimes \Pi_{h}\right) \operatorname{Cor}[\langle\mathbf{V}, \mathbf{n}\rangle]\right) .
$$

Then, replacing the energy space $H_{\text {mix }}^{-1 / 2,-1 / 2}\left(\partial D_{0} \times \partial D_{0}\right)$ by the finite dimensional ansatz space $S_{h}^{d} \otimes S_{h}^{d} \subset H_{\text {mix }}^{-1 / 2,-1 / 2}\left(\partial D_{0} \times \partial D_{0}\right)$, we end up with the system of linear equations

$$
(\mathbf{V} \otimes \mathbf{V}) \operatorname{vec}\left(\mathbf{C}_{\rho, \operatorname{Cor}[\delta u]}\right)=(\mathbf{N} \otimes \mathbf{N})(\mathbf{M} \otimes \mathbf{M})^{-1} \operatorname{vec}\left(\mathbf{C}_{\operatorname{Cor}[\langle\mathbf{V}, \mathbf{n}\rangle]}\right) .
$$

The corresponding matrices $\mathbf{N}$ and $\mathbf{C}_{\rho, \operatorname{Cor}[\delta u]}$ are given by

$$
\begin{aligned}
\mathbf{N} & =\left[\left(\left(\frac{\partial g}{\partial \mathbf{n}}-t_{h}^{\left(u_{0}\right)}\right) \psi_{j}, \psi_{i}\right)_{L^{2}\left(\partial D_{0}\right)}\right]_{i, j=1}^{n} \\
\mathbf{C}_{\rho, \operatorname{Cor}[\delta u]} & =\left[c_{i, j}^{\rho, \operatorname{Cor}[\delta u]}\right]_{i, j=1}^{n},
\end{aligned}
$$

where the coefficients $c_{i, j}^{\rho, \operatorname{Cor}[\delta u]}$ are the coefficients of the basis expansion

$$
\rho_{h}^{(\operatorname{Cor}[\delta u])}=\sum_{i, j=1}^{n} c_{i, j}^{\rho, \operatorname{Cor}[\delta u]}\left(\psi_{i} \otimes \psi_{j}\right) .
$$

Due to 24 , we can write 26] equivalently as

$$
\mathbf{V C}_{\rho, \operatorname{Cor}[\delta u]} \mathbf{V}^{\boldsymbol{\top}}=\mathbf{N M}^{-1} \mathbf{C}_{\operatorname{Cor}[\langle\mathbf{V}, \mathbf{n}\rangle]} \mathbf{M}^{-\top} \mathbf{N}^{\top} .
$$

\subsection{Computation of $\mathbb{E}\left[\delta^{2} u\right]$}

For the computation of $\mathbb{E}\left[\delta^{2} u\right]$, we shall first consider the numerical treatment of the computation of the correlation $A$ as given by $(19)$. Its variational formulation is:

Find $A \in H_{\text {mix }}^{0,-1 / 2}\left(\partial D_{0} \times \partial D_{0}\right)$ such that

$$
\begin{aligned}
& ((\operatorname{Id} \otimes \mathcal{V}) A, v)_{L^{2}\left(\partial D_{0} \times \partial D_{0}\right)}= \\
& \quad\left(\left(\operatorname{Id} \otimes\left(\frac{1}{2}+\mathcal{K}\right)\right)\left(\left(\mathbb{1} \otimes\left(\frac{\partial g}{\partial \mathbf{n}}-t^{\left(u_{0}\right)}\right)\right) \operatorname{Cor}[\langle\mathbf{V}, \mathbf{n}\rangle], v\right)_{L^{2}\left(\partial D_{0} \times \partial D_{0}\right)}\right.
\end{aligned}
$$

for all $v \in H_{\operatorname{mix}}^{0,-1 / 2}\left(\partial D_{0} \times \partial D_{0}\right)$.

To deal with the products in the right-hand side, we can use the same strategy as for the Dirichletto-Neumann map (15) and as for 26), i.e., by introducing $L^{2}$-projections. Then, replacing the 
energy space $H_{\text {mix }}^{0,-1 / 2}\left(\partial D_{0} \times \partial D_{0}\right)$ by the finite dimensional ansatz space $S_{h}^{d} \otimes S_{h}^{d} \subset H_{\text {mix }}^{0,-1 / 2}\left(\partial D_{0} \times\right.$ $\left.\partial D_{0}\right)$, we end up with the system of linear equations

$$
(\mathbf{M} \otimes \mathbf{V}) \operatorname{vec}(\mathbf{A})=\left(\mathbf{M} \otimes\left(\frac{1}{2} \mathbf{M}+\mathbf{K}\right)\right)(\mathbf{M} \otimes \mathbf{M})^{-1}(\mathbf{M} \otimes \mathbf{N})(\mathbf{M} \otimes \mathbf{M})^{-1} \operatorname{vec}\left(\mathbf{C}_{\operatorname{Cor}[\langle\mathbf{V}, \mathbf{n}\rangle]}\right),
$$

where $\mathbf{A}$ contains the coefficients of the basis expansion of the Galerkin solution $A_{h} \in S_{h}^{d} \otimes S_{h}^{d}$. Rearranging this system according to 24 yields the matrix equation

$$
\mathbf{V A M}^{\boldsymbol{\top}}=\left(\frac{1}{2} \mathbf{M}+\mathbf{K}\right) \mathbf{M}^{-1} \mathbf{N M}^{-1} \mathbf{C}_{\mathrm{Cor}[\langle\mathbf{V}, \mathbf{n}\rangle]} .
$$

Having approximated the correlation $A$, the Dirichlet data of $\mathbb{E}\left[\delta^{2} u\right]$ can now be computed. Following the considerations of the discretization of the Dirichlet-to-Neumann map from Section 4.1 we employ again $L^{2}$-projections to deal with the products of the right-hand side. The Neumann data $t^{\left(\mathbb{E}\left[\delta^{2} u\right]\right)}$ of $\mathbb{E}\left[\delta^{2} u\right]$ are then given by

$$
\mathbf{V} \mathbf{t}^{\left(\mathbb{E}\left[\delta^{2} u\right]\right)}=\left(\frac{1}{2} \mathbf{M}+\mathbf{K}\right) \mathbf{M}^{-1}\left(\left(\mathbf{G}-\mathbf{L} \mathbf{M}^{-1} \mathbf{N}\right) \mathbf{M}^{-1} \mathbf{d}-2 \mathbf{b}\right)
$$

with corresponding vectors and matrices

$$
\begin{array}{lll}
\mathbf{d}=\left[\left(\left.\operatorname{Cor}[\langle\mathbf{V}, \mathbf{n}\rangle]\right|_{\mathbf{x}=\mathbf{y}}, \psi_{i}\right)_{L^{2}\left(\partial D_{0}\right)}\right]_{i=1}^{n}, & \mathbf{b}=\left[\left(\left.A_{h}\right|_{\mathbf{x}=\mathbf{y}}, \psi_{i}\right)_{L^{2}\left(\partial D_{0}\right)}\right]_{i=1}^{n}, \\
\mathbf{L}=\left[\left(2 \mathcal{H} \psi_{j}, \psi_{i}\right)_{L^{2}\left(\partial D_{0}\right)}\right]_{i, j=1}^{n}, & \mathbf{G}=\left[\left(\Delta g \psi_{j}, \psi_{i}\right)_{L^{2}\left(\partial D_{0}\right)}\right]_{i, j=1}^{n} .
\end{array}
$$

The correction term $\mathbb{E}\left[\delta^{2} u\right]$ itself is then given by the representation formula (21).

The error estimation of the approximate solutions is the topic of the next section.

\section{Error Estimates}

For the following error estimates, it is important to carefully distinguish between the regularity requirements of the involved shape calculus and the regularity assumptions for the Galerkin discretization. More specifically, let us remark that a regularity assumption on the boundary $\partial D_{0}$ of the reference domain does not necessarily imply the same regularity to the perturbations and the perturbed domains $D(\omega)$. Throughout our proofs, we require that the domain $D_{0}$ is of class $C^{d, 1}$ and that the prescribed Dirichlet data satisfy $g \in H^{d+1}\left(\partial D_{0}\right)$.

\subsection{Preliminaries}

We start by restating a convergence result for the Dirichlet-to-Neumann map.

Lemma 5.1. For the Neumann data $t^{\left(u_{0}\right)}$ of the solution $u_{0}$ of the unperturbed problem (2) and their approximation $t_{h}^{\left(u_{0}\right)}$, it holds for $-d \leq s \leq 0$ that

$$
\left\|t^{\left(u_{0}\right)}-t_{h}^{\left(u_{0}\right)}\right\|_{H^{s}\left(\partial D_{0}\right)} \lesssim h^{d-s} .
$$

Proof. The proof for the case $-d \leq s \leq-1 / 2$ is standard, we refer to 28 and remark that $\mathcal{K}: H^{s}\left(\partial D_{0}\right) \rightarrow H^{s+1}\left(\partial D_{0}\right)$ is a continuous operator on $C^{d, 1}$-boundaries. To extend the result to the case $-1 / 2<s \leq 0$, consider the inequality

$$
\left\|t^{\left(u_{0}\right)}-t_{h}^{\left(u_{0}\right)}\right\|_{H^{s}\left(\partial D_{0}\right)} \leq\left\|t^{\left(u_{0}\right)}-\Pi_{h} t^{\left(u_{0}\right)}\right\|_{H^{s}\left(\partial D_{0}\right)}+\left\|\Pi_{h} t^{\left(u_{0}\right)}-t_{h}^{\left(u_{0}\right)}\right\|_{H^{s}\left(\partial D_{0}\right)} .
$$


The first term on the right hand side can again be estimated by the standard approximation property of the $L^{2}$-projection, whereas, for the second term on the right hand side, we employ the inverse estimate to obtain

$$
\begin{aligned}
\left\|\Pi_{h} t^{\left(u_{0}\right)}-t_{h}^{\left(u_{0}\right)}\right\|_{H^{s}\left(\partial D_{0}\right)} & \leq h^{s-1 / 2}\left\|\Pi_{h} t^{\left(u_{0}\right)}-t_{h}^{\left(u_{0}\right)}\right\|_{H^{-1 / 2}\left(\partial D_{0}\right)} \\
& \leq h^{s-1 / 2}\left(\left\|\Pi_{h} t^{\left(u_{0}\right)}-t^{\left(u_{0}\right)}\right\|_{H^{-1 / 2}\left(\partial D_{0}\right)}+\left\|t^{\left(u_{0}\right)}-t_{h}^{\left(u_{0}\right)}\right\|_{H^{-1 / 2}\left(\partial D_{0}\right)}\right) .
\end{aligned}
$$

This yields the assertion together with the first part of the lemma and the standard approximation estimates of the $L^{2}$-projection.

The following technical lemma is needed for the error estimation and is inspired in parts by the proof of [7, Theorem 7.3].

Lemma 5.2. Assume that $a \in L^{2}\left(\partial D_{0}\right), b \in H^{d}\left(\partial D_{0}\right)$ and that the numerical approximation $a_{h}$ of a satisfies $\left\|a-a_{h}\right\|_{L^{2}\left(\partial D_{0}\right)} \lesssim h^{d}$, and $\left\|a-a_{h}\right\|_{H^{s}\left(\partial D_{0}\right)} \lesssim h^{d-s}$ for $-d \leq s<-(n-1) / 2$. Then, we have

$$
\left\|a b-a_{h} \Pi_{h} b\right\|_{H^{s}\left(\partial D_{0}\right)} \lesssim h^{d-s}
$$

Proof. We start by splitting the error into two parts

$$
\left\|a b-a_{h} \Pi_{h} b\right\|_{H^{s}\left(\partial D_{0}\right)} \leq\left\|\left(a-a_{h}\right) b\right\|_{H^{s}\left(\partial D_{0}\right)}+\left\|a_{h}\left(\mathrm{Id}-\Pi_{h}\right) b\right\|_{H^{s}\left(\partial D_{0}\right)} .
$$

For $u, v \in H^{-s}\left(\partial D_{0}\right)$, we have the estimate $\|u v\|_{H^{-s}\left(\partial D_{0}\right)} \lesssim\|u\|_{H^{-s}\left(\partial D_{0}\right)}\|v\|_{H^{-s}\left(\partial D_{0}\right)}$, cf. [29, 30], Thus, we conclude by duality that it holds for $u \in H^{s}\left(\partial D_{0}\right)$ and $v \in H^{-s}\left(\partial D_{0}\right)$ that

$$
\begin{aligned}
\|u v\|_{H^{s}\left(\partial D_{0}\right)} & =\sup _{\|w\|_{H^{-s}\left(\partial D_{0}\right)}=1}(u v, w)_{L^{2}\left(\partial D_{0}\right)} \\
& =\sup _{\|w\|_{H^{-s}\left(\partial D_{0}\right)}=1}(u, v w)_{L^{2}\left(\partial D_{0}\right)} \\
& \leq\|u\|_{H^{s}\left(\partial D_{0}\right)} \sup _{\|w\|_{H^{-s}\left(\partial D_{0}\right)}=1}\|v w\|_{H^{-s}\left(\partial D_{0}\right)} \\
& \lesssim\|u\|_{H^{s}\left(\partial D_{0}\right)}\|v\|_{H^{-s}\left(\partial D_{0}\right)}
\end{aligned}
$$

Thus, the first part of the error can easily be estimated by

$$
\left\|\left(a-a_{h}\right) b\right\|_{H^{s}\left(\partial D_{0}\right)} \lesssim\left\|a-a_{h}\right\|_{H^{s}\left(\partial D_{0}\right)}\|b\|_{H^{-s}\left(\partial D_{0}\right)},
$$

whereas the second part of the error is treated in accordance with

$$
\begin{aligned}
& \left\|a_{h}\left(\mathrm{Id}-\Pi_{h}\right) b\right\|_{H^{s}\left(\partial D_{0}\right)} \\
& \quad=\sup _{\|w\|_{H^{-s}\left(\partial D_{0}\right)}=1}\left(a_{h}\left(\mathrm{Id}-\Pi_{h}\right) b, w\right)_{L^{2}\left(\partial D_{0}\right)} \\
& \quad=\sup _{\|w\|_{H^{-s}\left(\partial D_{0}\right)}=1}\left(\left(\left(a_{h}-a\right)\left(\mathrm{Id}-\Pi_{h}\right) b, w\right)_{L^{2}\left(\partial D_{0}\right)}+\left(a\left(\operatorname{Id}-\Pi_{h}\right) b, w\right)_{L^{2}\left(\partial D_{0}\right)}\right) .
\end{aligned}
$$

Since $H^{-s}\left(\partial D_{0}\right)$ is continuously embedded into $L^{\infty}\left(\partial D_{0}\right)$ on $C^{d, 1}$-smooth boundaries for $-s>$ $(n-1) / 2$, one can estimate

$$
\begin{aligned}
\left(\left(a_{h}-a\right)\left(\mathrm{Id}-\Pi_{h}\right) b, w\right)_{L^{2}\left(\partial D_{0}\right)} & \leq\left\|\left(a_{h}-a\right)\left(\mathrm{Id}-\Pi_{h}\right) b\right\|_{L^{1}\left(\partial D_{0}\right)}\|w\|_{L^{\infty}\left(\partial D_{0}\right)} \\
& \lesssim\left\|a-a_{h}\right\|_{L^{2}\left(\partial D_{0}\right)}\left\|\left(\mathrm{Id}-\Pi_{h}\right) b\right\|_{L^{2}\left(\partial D_{0}\right)}\|w\|_{H^{-s}\left(\partial D_{0}\right)}
\end{aligned}
$$


and, by employing Galerkin orthogonality,

$$
\begin{aligned}
\left(a\left(\mathrm{Id}-\Pi_{h}\right) b, w\right)_{L^{2}\left(\partial D_{0}\right)} & =\left(\left(\mathrm{Id}-\Pi_{h}\right) b, a w\right)_{L^{2}\left(\partial D_{0}\right)} \\
& =\left(\left(\mathrm{Id}-\Pi_{h}\right) b,\left(\mathrm{Id}-\Pi_{h}\right)(a w)\right)_{L^{2}\left(\partial D_{0}\right)} \\
& \leq\left\|\left(\operatorname{Id}-\Pi_{h}\right) b\right\|_{L^{2}\left(\partial D_{0}\right)}\left\|\left(\mathrm{Id}-\Pi_{h}\right)(a w)\right\|_{L^{2}\left(\partial D_{0}\right)} .
\end{aligned}
$$

The assertion follows by applying the standard approximation estimates of the $L^{2}$-projection, implying that the first part of product is bounded by $\lesssim h^{d}$ and the second part of product is bounded by $\lesssim h^{-s}$.

By a tensor product argument, we arrive at a similar statement on the tensor product domain.

Corollary 5.3. Assume that $a \in L^{2}\left(\partial D_{0} \times \partial D_{0}\right)$ and $b \in H_{\text {mix }}^{d, d}\left(\partial D_{0} \times \partial D_{0}\right)$ and that the numerical approximation $a_{h}$ of a satisfies $\left\|a-a_{h}\right\|_{L^{2}\left(\partial D_{0} \times \partial D_{0}\right)} \lesssim h^{d}$, and $\left\|a-a_{h}\right\|_{H_{\text {mix }}^{s, s}\left(\partial D_{0} \times \partial D_{0}\right)} \lesssim h^{d-s}$ for $-d \leq s<-(n-1) / 2$. Then, it holds that

$$
\left\|a b-a_{h}\left(\Pi_{h} \otimes \Pi_{h}\right) b\right\|_{H_{\text {mix }}^{s, s}\left(\partial D_{0} \times \partial D_{0}\right)} \lesssim h^{d-s} .
$$

Proof. The proof is very similar to the proof of the preceding lemma. We refer to the proof of [7, Theorem 7.3], where the details are given for the case $s=-d$, using the estimate $\|u v\|_{H^{d}\left(\partial D_{0}\right)} \leq$ $\|u\|_{C^{d-1,1}\left(\partial D_{0}\right)}\|v\|_{H^{d}\left(\partial D_{0}\right)}$ instead of $\|u v\|_{H^{d}\left(\partial D_{0}\right)} \lesssim\|u\|_{H^{d}\left(\partial D_{0}\right)}\|v\|_{H^{d}\left(\partial D_{0}\right)}$.

Remark 5.4. The focus of the present article is on the cases $n=2,3$ such that $(n-1) / 2<d$ implies $d \geq 1$ for $n=2$ and $d \geq 2$ for $n=3$, respectively. To simplify the presentation of the following results, we will therefore restrict ourselves to the case $d \geq 2$, i.e., we are considering discretizations with at least piecewise linear continuous ansatz functions.

\subsection{Approximation Error of $\operatorname{Cor}[\delta u]$}

In order to bound the approximation error of Cor $[\delta u]$ given by the representation formula (23), we first have to bound the approximation error of its boundary values. This is in parts due to the following lemma.

Lemma 5.5. Let $\operatorname{Cor}[\langle\mathbf{V}, \mathbf{n}\rangle] \in H_{\text {mix }}^{d, d}\left(\partial D_{0} \times \partial D_{0}\right)$. Then, it holds

$$
\left\|g^{(\operatorname{Cor}[\delta u])}-g_{h}^{(\operatorname{Cor}[\delta u])}\right\|_{H_{\text {mix }}^{-d,-d}\left(\partial D_{0} \times \partial D_{0}\right)} \lesssim h^{2 d} .
$$

Proof. The assertion is an immediate consequence of Corollary 5.3 with

$$
a=\left(\frac{\partial g}{\partial \mathbf{n}}-t^{\left(u_{0}\right)}\right) \otimes\left(\frac{\partial g}{\partial \mathbf{n}}-t^{\left(u_{0}\right)}\right), \quad a_{h}=\left(\frac{\partial g}{\partial \mathbf{n}}-t_{h}^{\left(u_{0}\right)}\right) \otimes\left(\frac{\partial g}{\partial \mathbf{n}}-t_{h}^{\left(u_{0}\right)}\right),
$$

and $b=\operatorname{Cor}[\langle\mathbf{V}, \mathbf{n}\rangle]$. The required convergence in the $H^{-d}\left(\partial D_{0}\right)$-norm and in the $L^{2}\left(\partial D_{0}\right)$-norm is proven in Lemma 5.1 for the non-tensor product case. For the tensor product case, consider for $-d \leq s \leq 0$ that

$$
\begin{aligned}
\| a- & a_{h} \|_{H_{\text {mix }}^{s, s}\left(\partial D_{0} \times \partial D_{0}\right)} \\
& =\left\|\left(\frac{\partial g}{\partial \mathbf{n}}-t^{\left(u_{0}\right)}\right) \otimes\left(t_{h}^{\left(u_{0}\right)}-t^{\left(u_{0}\right)}\right)+\left(t_{h}^{\left(u_{0}\right)}-t^{\left(u_{0}\right)}\right) \otimes\left(\frac{\partial g}{\partial \mathbf{n}}-t_{h}^{\left(u_{0}\right)}\right)\right\|_{H_{\text {mix }}^{s, s}\left(\partial D_{0} \times \partial D_{0}\right)} \\
& \leq\left\|\frac{\partial g}{\partial \mathbf{n}}-t^{\left(u_{0}\right)}\right\|_{H^{s}\left(\partial D_{0}\right)}\left\|t^{\left(u_{0}\right)}-t_{h}^{\left(u_{0}\right)}\right\|_{H^{s}\left(\partial D_{0}\right)}+\left\|t^{\left(u_{0}\right)}-t_{h}^{\left(u_{0}\right)}\right\|_{H^{s}\left(\partial D_{0}\right)}\left\|\frac{\partial g}{\partial \mathbf{n}}-t_{h}^{\left(u_{0}\right)}\right\|_{H^{s}\left(\partial D_{0}\right)} \\
& \lesssim h^{d-s .}
\end{aligned}
$$

This completes the proof. 
We are finally in the position to estimate the error of $\operatorname{Cor}[\delta u]_{h}$.

Lemma 5.6. Let $\operatorname{Cor}[\delta u]$ be given as in 23 and let $\operatorname{Cor}[\langle\mathbf{V}, \mathbf{n}\rangle] \in H_{\text {mix }}^{d, d}\left(\partial D_{0} \times \partial D_{0}\right)$. It then holds

$$
\left|\operatorname{Cor}[\delta u](\mathbf{x}, \mathbf{y})-\operatorname{Cor}[\delta u]_{h}(\mathbf{x}, \mathbf{y})\right| \lesssim h^{2 d} \quad \text { for all } \mathbf{x}, \mathbf{y} \in K \Subset D_{\cap \Omega} .
$$

Proof. By employing the representation formula (23), we obtain

$$
\begin{aligned}
&\left|\operatorname{Cor}[\delta u](\mathbf{x}, \mathbf{y})-\operatorname{Cor}[\delta u]_{h}(\mathbf{x}, \mathbf{y})\right| \\
& \quad=\left|(\tilde{\mathcal{V}} \otimes \tilde{\mathcal{V}})\left(\rho^{(\operatorname{Cor}[\delta u])}-\rho_{h}^{(\operatorname{Cor}[\delta u])}\right)(\mathbf{x}, \mathbf{y})\right| \\
& \lesssim\left\|\frac{1}{16 \pi^{2}\|\mathbf{x}-\cdot\| \otimes\|\mathbf{y}-\cdot\|}\right\|\left\|_{H_{\text {mix }}^{d+1, d+1}\left(\partial D_{0} \times \partial D_{0}\right)}\right\| \rho^{(\operatorname{Cor}[\delta u])}-\rho_{h}^{(\operatorname{Cor}[\delta u])} \|_{H_{\text {mix }}^{-d-1,-d-1}\left(\partial D_{0} \times \partial D_{0}\right)} .
\end{aligned}
$$

Using Strang's first lemma and Lemma 5.5, we further derive

$$
\left\|\rho^{(\operatorname{Cor}[\delta u])}-\rho_{h}^{(\operatorname{Cor}[\delta u])}\right\|_{H_{\text {mix }}^{-d-1,-d-1}\left(\partial D_{0} \times \partial D_{0}\right)} \lesssim h^{2 d}
$$

which, in view of $K \Subset \partial D_{0}$ and thus $\operatorname{dist}\left(K, \partial D_{0}\right)>0$, implies the assertion.

Remark 5.7. The assumptions of the presented result are slightly weaker than the related result from [7]. Whereas [7] requires $\operatorname{Cor}[\langle\mathbf{V}, \mathbf{n}\rangle]$ to be in $C_{\operatorname{mix}}^{d-1,1}\left(\partial D_{0} \times \partial D_{0}\right)$, we only require it to be in $H_{\text {mix }}^{d, d}\left(\partial D_{0} \times \partial D_{0}\right)$. This means that we do not need any extra regularity of the boundary perturbation in addition to the regularity required for the first order shape derivative and the Galerkin approximation of $\operatorname{Cor}[\langle\mathbf{V}, \mathbf{n}\rangle]$.

\subsection{Approximation Error of $\mathbb{E}\left[\delta^{2} u\right]$}

In order to estimate the discretization error of $\mathbb{E}\left[\delta^{2} u\right]$ given by $(13)$, we need to know the discretization error of its Dirichlet data. Especially, we need to estimate the error of the correlation $A$ as given by 19 .

Lemma 5.8. Let $\operatorname{Cor}[\langle\mathbf{V}, \mathbf{n}\rangle] \in H_{\text {mix }}^{d, d}\left(\partial D_{0} \times \partial D_{0}\right)$. It then holds for

$$
A_{h}=\left(\operatorname{Id} \otimes \mathcal{V}^{-1}\right)\left(\operatorname{Id} \otimes\left(\frac{1}{2}+\mathcal{K} \Pi_{h}\right)\right)\left(\left(\mathbb{1} \otimes\left(\frac{\partial g}{\partial \mathbf{n}}-t_{h}^{\left(u_{0}\right)}\right)\right)\left(\Pi_{h} \otimes \Pi_{h}\right) \operatorname{Cor}[\langle\mathbf{V}, \mathbf{n}\rangle]\right)
$$

that

Proof. For

$$
\left\|A-A_{h}\right\|_{H_{\mathrm{mix}}^{-d+1,-d+1}\left(\partial D_{0} \times \partial D_{0}\right)} \lesssim h^{2(d-1)} .
$$

$$
a=\mathbb{1} \otimes\left(\frac{\partial g}{\partial \mathbf{n}}-t^{\left(u_{0}\right)}\right), \quad a_{h}=\mathbb{1} \otimes\left(\frac{\partial g}{\partial \mathbf{n}}-t_{h}^{\left(u_{0}\right)}\right), \quad b=\operatorname{Cor}[\langle\mathbf{V}, \mathbf{n}\rangle]
$$

it holds

$$
\begin{aligned}
& \left\|A-A_{h}\right\|_{H_{\text {mix }}^{-d+1,-d+1}\left(\partial D_{0} \times \partial D_{0}\right)} \\
& \leq\left\|\operatorname{Id} \otimes \mathcal{V}^{-1}\right\|_{H_{\text {mix }}^{-d+2,-d+2}\left(\partial D_{0} \times \partial D_{0}\right) \rightarrow H_{\text {mix }}^{-d+1,-d+1}\left(\partial D_{0} \times \partial D_{0}\right)} \\
& \left\|\left(\mathrm{Id} \otimes\left(\frac{1}{2}+\mathcal{K}\right)\right)(a b)-\left(\mathrm{Id} \otimes\left(\frac{1}{2}+\mathcal{K} \Pi_{h}\right)\right)\left(a_{h}\left(\Pi_{h} \otimes \Pi_{h}\right) b\right)\right\|_{H_{\text {mix }}^{-d+2,-d+2}\left(\partial D_{0} \times \partial D_{0}\right)} \\
& \lesssim\|\operatorname{Id} \otimes \mathcal{K}\|_{H_{\text {mix }}^{-d+2,-d+2}\left(\partial D_{0} \times \partial D_{0}\right) \rightarrow H_{\text {mix }}^{-d+2,-d+2}\left(\partial D_{0} \times \partial D_{0}\right)} \\
& \left\|a b-\left(\operatorname{Id} \otimes \Pi_{h}\right)(a b)\right\|_{H_{\text {mix }}^{-d+2,-d+2}\left(\partial D_{0} \times \partial D_{0}\right)} \\
& +\left\|\mathrm{Id} \otimes\left(\frac{1}{2}+\mathcal{K} \Pi_{h}\right)\right\|_{H_{\text {mix }}^{-d+2,-d+2}\left(\partial D_{0} \times \partial D_{0}\right) \rightarrow H_{\text {mix }}^{-d+2,-d+2}\left(\partial D_{0} \times \partial D_{0}\right)} \\
& \left\|a b-a_{h}\left(\Pi_{h} \otimes \Pi_{h}\right) b\right\|_{H_{\text {mix }}^{-d+2,-d+2}\left(\partial D_{0} \times \partial D_{0}\right)} .
\end{aligned}
$$


The assertion now follows from the approximation property of the $L^{2}$-projection, Lemma 5.1 , and Corollary 5.3 .

The previous lemma allows us to bound the error of the Dirichlet data of the boundary value problem 13 for $\mathbb{E}\left[\delta^{2} u\right]$.

Lemma 5.9. Let $\left.\operatorname{Cor}[\langle\mathbf{V}, \mathbf{n}\rangle]\right|_{\mathbf{x}=\mathbf{y}} \in H^{d}\left(\partial D_{0}\right)$ and $\operatorname{Cor}[\langle\mathbf{V}, \mathbf{n}\rangle] \in H_{\operatorname{mix}}^{d, d}\left(\partial D_{0} \times \partial D_{0}\right)$. For the Dirichlet data of $\mathbb{E}\left[\delta^{2} u\right]$ and their numerical approximation

$$
g_{h}^{\left(\mathbb{E}\left[\delta^{2} u\right]\right)}=\left(\Delta g-2 \mathcal{H} \Pi_{h}\left(\frac{\partial g}{\partial \mathbf{n}}-t_{h}^{\left(u_{0}\right)}\right)\right) \Pi_{h}\left(\left.\operatorname{Cor}[\langle\mathbf{V}, \mathbf{n}\rangle]\right|_{\mathbf{x}=\mathbf{y}}\right)-\left.2 A_{h}\right|_{\mathbf{x}=\mathbf{y}},
$$

it holds

$$
\left\|g^{\left(\mathbb{E}\left[\delta^{2} u\right]\right)}-g_{h}^{\left(\mathbb{E}\left[\delta^{2} u\right]\right)}\right\|_{H^{-d}\left(\partial D_{0}\right)} \lesssim h^{2(d-1)}
$$

and

$$
\left\|g^{\left(\mathbb{E}\left[\delta^{2} u\right]\right)}-\Pi_{h} g_{h}^{\left(\mathbb{E}\left[\delta^{2} u\right]\right)}\right\|_{H^{-d}\left(\partial D_{0}\right)} \lesssim h^{2(d-1)} .
$$

Proof. By exploiting $\Pi_{h} t_{h}^{\left(u_{0}\right)}=t_{h}^{\left(u_{0}\right)}$ and setting

$$
a=\frac{\partial g}{\partial \mathbf{n}}-t^{\left(u_{0}\right)}, \quad a_{h}=\Pi_{h} \frac{\partial g}{\partial \mathbf{n}}-t_{h}^{\left(u_{0}\right)}, \quad b=\left.\operatorname{Cor}[\langle\mathbf{V}, \mathbf{n}\rangle]\right|_{\mathbf{x}=\mathbf{y}}
$$

we conclude

$$
\begin{aligned}
\left\|g^{\left(\mathbb{E}\left[\delta^{2} u\right]\right)}-g_{h}^{\left(\mathbb{E}\left[\delta^{2} u\right]\right)}\right\|_{H^{-d}\left(\partial D_{0}\right)} \leq\left\|(\Delta g)\left(b-\Pi_{h} b\right)\right\|_{H^{-d}\left(\partial D_{0}\right)} \\
\quad+\left\|2 \mathcal{H}\left(a b-a_{h} \Pi_{h} b\right)\right\|_{H^{-d}\left(\partial D_{0}\right)}+2\left\|\left.A\right|_{\mathbf{x}=\mathbf{y}}-\left.A_{h}\right|_{\mathbf{x}=\mathbf{y}}\right\|_{H^{-d}\left(\partial D_{0}\right)}
\end{aligned}
$$

Herein, the first term on the right hand side can be bounded by $\lesssim h^{2 d}$ by applying the standard approximation estimates of the $L^{2}$-projection. The second term on the right hand can be estimated by using the inequality $\|u v\|_{H^{-d+2}\left(\partial D_{0}\right)} \lesssim\|u\|_{H^{d-2}\left(\partial D_{0}\right)}\|v\|_{H^{-d+2}\left(\partial D_{0}\right)}$ derived in the proof of Lemma 5.2.

$$
\begin{aligned}
\left\|2 \mathcal{H}\left(a b-a_{h} \Pi_{h} b\right)\right\|_{H^{-d}\left(\partial D_{0}\right)} & \leq\left\|2 \mathcal{H}\left(a b-a_{h} \Pi_{h} b\right)\right\|_{H^{-d+2}\left(\partial D_{0}\right)} \\
& \lesssim\|2 \mathcal{H}\|_{H^{d-2}\left(\partial D_{0}\right)}\left\|a b-a_{h} \Pi_{h} b\right\|_{H^{-d+2}\left(\partial D_{0}\right)}
\end{aligned}
$$

To estimate the third term on the right hand side, we follow the arguments in [31, Chapter 3.3] and remark that the diagonal operator $H_{\text {mix }}^{s, s}\left(\partial D_{0} \times \partial D_{0}\right) \rightarrow W^{s, 1}\left(\partial D_{0}\right)$ is continuous for $s \geq 1$. Exploiting that the embedding $W^{s, 1}\left(\partial D_{0}\right) \hookrightarrow H^{s-1}\left(\partial D_{0}\right)$ is also continuous, we conclude by a density argument that

$$
\left\|\left.A\right|_{\mathbf{x}=\mathbf{y}}-\left.A_{h}\right|_{\mathbf{x}=\mathbf{y}}\right\|_{H^{-d}\left(\partial D_{0}\right)} \lesssim\left\|A-A_{h}\right\|_{H_{\text {mix }}^{-d+1,-d+1}\left(\partial D_{0} \times \partial D_{0}\right)} .
$$

In view of Lemmata 5.2 and 5.8 this implies the estimate 30 .

Estimate (31) follows finally from (30) and Strang's first lemma by considering the Galerkin projection as the solution of $\operatorname{Id} \psi=g$ with a perturbed right-hand side.

We are now in the position to bound the error of the Neumann data $t^{\left(\mathbb{E}\left[\delta^{2} u\right]\right)}$ of $\mathbb{E}\left[\delta^{2} u\right]$.

Lemma 5.10. Let $\left.\operatorname{Cor}[\langle\mathbf{V}, \mathbf{n}\rangle]\right|_{\mathbf{x}=\mathbf{y}} \in H^{d}\left(\partial D_{0}\right)$ and $\operatorname{Cor}[\langle\mathbf{V}, \mathbf{n}\rangle] \in H_{\text {mix }}^{d, d}\left(\partial D_{0} \times \partial D_{0}\right)$. Then, the Neumann data $t^{\left(\mathbb{E}\left[\delta^{2} u\right]\right)}$ are approximated with the rate

$$
\left\|t^{\left(\mathbb{E}\left[\delta^{2} u\right]\right)}-t_{h}^{\left(\mathbb{E}\left[\delta^{2} u\right]\right)}\right\|_{H^{-d-1}\left(\partial D_{0}\right)} \lesssim h^{2(d-1)}
$$


Proof. Since $\mathcal{V}: H^{d}\left(\partial D_{0}\right) \rightarrow H^{d+1}\left(\partial D_{0}\right)$ is self-adjoint, continuous, and boundedly invertible in case of a $C^{d, 1}$-boundary, it holds

$$
\begin{aligned}
\left\|t^{\left(u_{0}\right)}-t_{h}^{\left(u_{0}\right)}\right\|_{H^{-d-1}\left(\partial D_{0}\right)} & =\sup _{\|v\|_{H^{d+1}\left(\partial D_{0}\right)}=1}\left(t^{\left(u_{0}\right)}-t_{h}^{\left(u_{0}\right)}, v\right)_{L^{2}\left(\partial D_{0}\right)} \\
& =\sup _{\|w\|_{H^{d}\left(\partial D_{0}\right)}=1} \frac{\left(\mathcal{V}\left(t^{\left(u_{0}\right)}-t_{h}^{\left(u_{0}\right)}\right), w\right)_{L^{2}\left(\partial D_{0}\right)}}{\|\mathcal{V} w\|_{H^{d+1}\left(\partial D_{0}\right)}} \\
\leq & \sup _{\|w\|_{H^{d}\left(\partial D_{0}\right)}} \frac{\left(\mathcal{V}\left(t^{\left(u_{0}\right)}-t_{h}^{\left(u_{0}\right)}\right), w-\Pi_{h} w\right)_{L^{2}\left(\partial D_{0}\right)}}{\|\mathcal{V} w\|_{H^{d+1}\left(\partial D_{0}\right)}} \\
& +\sup _{\|w\|_{H^{d}\left(\partial D_{0}\right)}=1} \frac{\left(\mathcal{V}\left(t^{\left(u_{0}\right)}-t_{h}^{\left(u_{0}\right)}\right), \Pi_{h} w\right)_{L^{2}\left(\partial D_{0}\right)}}{\|\mathcal{V} w\|_{H^{d+1}\left(\partial D_{0}\right)}} .
\end{aligned}
$$

Using the continuity and bounded invertibility of $\mathcal{V}$, we further estimate

$$
\begin{aligned}
\sup _{\|w\|_{H^{d}\left(\partial D_{0}\right)}=1} & \frac{\left(\mathcal{V}\left(t^{\left(u_{0}\right)}-t_{h}^{\left(u_{0}\right)}\right), w-\Pi_{h} w\right)_{L^{2}\left(\partial D_{0}\right)}}{\|\mathcal{V} w\|_{H^{d+1}\left(\partial D_{0}\right)}} \\
& \leq\left\|\mathcal{V}\left(t^{\left(u_{0}\right)}-t_{h}^{\left(u_{0}\right)}\right)\right\|_{H^{1 / 2}\left(\partial D_{0}\right)} \sup _{\|w\|_{H^{d}\left(\partial D_{0}\right)}=1} \frac{\left\|w-\Pi_{h} w\right\|_{H^{-1 / 2}\left(\partial D_{0}\right)}}{\|\mathcal{V} w\|_{H^{d+1}\left(\partial D_{0}\right)}} \\
& \lesssim\left\|t^{\left(u_{0}\right)}-t_{h}^{\left(u_{0}\right)}\right\|_{H^{-1 / 2}\left(\partial D_{0}\right)\|w\|_{H^{d}\left(\partial D_{0}\right)}=1} \frac{\left\|w-\Pi_{h} w\right\|_{H^{-1 / 2}\left(\partial D_{0}\right)}}{\|w\|_{H^{d}\left(\partial D_{0}\right)}}
\end{aligned}
$$

Using standard error estimates for the Galerkin method, the error of the Neumann data in the $H^{-1 / 2}\left(\partial D_{0}\right)$-norm is bounded by $\lesssim h^{d+1 / 2}$, whereas the second factor can be estimated by the standard error estimates for the $L^{2}$-projection.

To estimate the second part of the sum, consider

$$
\left(\mathcal{V}\left(t^{\left(u_{0}\right)}-t_{h}^{\left(u_{0}\right)}\right), v_{h}\right)_{L^{2}\left(\partial D_{0}\right)}=\left(\frac{1}{2}\left(g-g_{h}\right)+\mathcal{K}\left(g-\Pi_{h} g_{h}\right), v_{h}\right)_{L^{2}\left(\partial D_{0}\right)} \quad \text { for all } v_{h} \in S_{h}^{d},
$$

and conclude

$$
\begin{aligned}
&\left(\mathcal { V } \left(t^{\left(u_{0}\right)}-\right.\right.\left.\left.t_{h}^{\left(u_{0}\right)}\right), \Pi_{h} w\right)_{L^{2}\left(\partial D_{0}\right)} \\
&=\left(\frac{1}{2}\left(g-g_{h}\right)+\mathcal{K}\left(g-\Pi_{h} g_{h}\right), \Pi_{h} w\right)_{L^{2}\left(\partial D_{0}\right)} \\
&=\left(\frac{1}{2}\left(g-g_{h}\right)+\mathcal{K}\left(g-\Pi_{h} g_{h}\right), w\right)_{L^{2}\left(\partial D_{0}\right)}+\frac{1}{2}\left(g-g_{h}, \Pi_{h} w-w\right)_{L^{2}\left(\partial D_{0}\right)} \\
& \quad+\left(\mathcal{K}\left(g-\Pi_{h} g_{h}\right), \Pi_{h} w-w\right)_{L^{2}\left(\partial D_{0}\right)}+\|\|_{H^{d}\left(\partial D_{0}\right)} \\
& \quad\left(\left\|g-g_{h}\right\|_{H-d}\left(\partial D_{0}\right)+\left\|\mathcal{K}\left(g-\Pi_{h} g_{h}\right)\right\|_{H^{-d}\left(\partial D_{0}\right)}\right)\|w\|_{H^{1}\left(\partial D_{0}\right)}\left\|w-\Pi_{h} w\right\|_{H^{-1}\left(\partial D_{0}\right)} \\
& \quad+\left\|\mathcal{K}\left(g-\Pi_{h} g_{h}\right)\right\|_{L^{2}\left(\partial D_{0}\right)}\left\|w-\Pi_{h} w\right\|_{L^{2}\left(\partial D_{0}\right)} . \\
& \quad+\left\|g-g_{h}\right\|_{L^{2}}
\end{aligned}
$$

The assertion now follows by exploiting that $\mathcal{K}: H^{s}\left(\partial D_{0}\right) \rightarrow H^{s}\left(\partial D_{0}\right)$ is a continuous operator for $s=-d$ and $s=1$ on $C^{d, 1}$-boundaries, the previous lemma, and inverse estimate in complete analogy to the proof of Lemma 5.1 . 
As an immediate consequence of the error estimates, we finally obtain an error estimate for the potential of the second order local shape derivative $\mathbb{E}\left[\delta^{2} u\right]$. The following approximation result can be derived by standard arguments, see [28] for instance.

Lemma 5.11. Let $\left.\operatorname{Cor}[\langle\mathbf{V}, \mathbf{n}\rangle]\right|_{\mathbf{x}=\mathbf{y}} \in H^{d}\left(\partial D_{0}\right)$ and $\operatorname{Cor}[\langle\mathbf{V}, \mathbf{n}\rangle] \in H_{\text {mix }}^{d, d}\left(\partial D_{0} \times \partial D_{0}\right)$. Then, for the mean of the second order local shape derivative $\mathbb{E}\left[\delta^{2} u\right]$ from $[13$, it holds that

$$
\left|\mathbb{E}\left[\delta^{2} u\right](\mathbf{x})-\mathbb{E}\left[\delta^{2} u\right]_{h}(\mathbf{x})\right| \lesssim h^{2(d-1)} \quad \text { for all } \mathbf{x} \in K \Subset D_{\cap \Omega} .
$$

Remark 5.12. Although we can only prove a reduced convergence rate of $h^{2(d-1)}$, we will see in the numerical experiments that we reach the same convergence rate as for the correlation, i.e., $h^{2 d}$.

\section{Hierarchical Matrix Compression of Dense Matrices}

Depending on the properties of the underlying correlation kernel, correlation matrices are usually densely populated. Similarly, it is well known from the numerical treatment of boundary integral equations that the Dirichlet-to-Neumann map leads to dense system matrices of the single layer operator (16) and the double layer operator (17). Although both operators live on the boundary $\partial D_{0}$, and have thus the spatial dimension reduced by one compared to $D_{0}$, the memory requirements and assembly time still scale at least like $\mathcal{O}\left(N^{2}\right)$, if ansatz spaces with dimension $N$ are used. To reduce the complexity to linear or almost linear (i.e. linear up to polylogarithmic terms) scaling in $N$, fast boundary element methods such as the fast multipole method 32, the panel clustering method [33, the wavelet Galerkin scheme [34, 35], and the adaptive cross approximation [36] exploit analytic properties of the kernel function to derive (data-)sparse approximations to the dense system matrices. Except for the wavelet Galerkin scheme, all theses methods can be cast into the framework of hierarchical matrices, cf. [37, 38, which we are going to employ throughout the rest of the article.

\subsection{Asymptotically Smooth Kernels}

We discuss the compression of the boundary operator matrices of the Dirichlet-to-Neumann map in a terms of the more general asymptotic smooth asymptotically smooth kernels.

Definition 6.1. The function $k: \mathbb{R}^{n} \times \mathbb{R}^{n} \rightarrow \mathbb{R}$ is called asymptotically smooth if for some constants $c_{1}, c_{2}>0$ and $q \in \mathbb{R}$ there holds

$$
\left|\partial_{\mathbf{x}}^{\boldsymbol{\alpha}} \partial_{\mathbf{y}}^{\boldsymbol{\beta}} k(\mathbf{x}, \mathbf{y})\right| \leq c_{1} \frac{(|\boldsymbol{\alpha}|+|\boldsymbol{\beta}|) !}{c_{2}^{|\boldsymbol{\alpha}|+|\boldsymbol{\beta}|}}\|\mathbf{x}-\mathbf{y}\|_{2}^{-n-2 q-|\boldsymbol{\alpha}|-|\boldsymbol{\beta}|}, \quad \mathbf{x} \neq \mathbf{y}
$$

independently of $\boldsymbol{\alpha}$ and $\boldsymbol{\beta}$. It is called asymptotically smooth in one variable, if the above condition holds only for the derivatives in $\mathbf{x}$ or $\mathbf{y}$.

We note especially that, besides the kernel functions of the single layer and the double layer operator, the widely used correlation kernels of the Matérn class, which include, in particular, the Gaussian kernel, cf. 39, 40, and the references therein, are asymptotically smooth or asymptotically smooth in one variable. We will therefore restrict ourselves to kernel functions of this type for the rest of this article and will exploit that they exhibit a data-sparse representation by means of hierarchial matrices ( $\mathcal{H}$-matrices in short).

$\mathcal{H}$-matrices rely on local low-rank approximations of a given matrix $\mathbf{X} \in \mathbb{R}^{N \times N}$. For suitable nonempty index sets $\nu, \nu^{\prime} \subset\{1, \ldots, N\}$, a matrix block $\left.\mathbf{X}\right|_{\nu \times \nu^{\prime}}$ can be approximated by a rank- $k$ matrix. This approximation can be represented in factorized form $\left.\mathbf{X}\right|_{\nu \times \nu^{\prime}} \approx \mathbf{Y} \mathbf{Z}^{\top}$ with factors $\mathbf{Y} \in \mathbb{R}^{\nu \times k}$ and $\mathbf{Z} \in \mathbb{R}^{\nu^{\prime} \times k}$. Hence, if $k \ll \min \left\{\# \nu, \# \nu^{\prime}\right\}$, the complexity for storing the block is considerably reduced. The construction of the index sets is based on the cluster tree. 


\subsection{Cluster Tree}

For a tree $\mathcal{T}=(V, E)$ with vertices $V$ and edges $E$, we define its set of leaves by

$$
\mathcal{L}(\mathcal{T}):=\{\sigma \in V: \sigma \text { has no sons }\} .
$$

Furthermore, we say that $\mathcal{T}$ is a cluster tree for the set $\{1, \ldots, N\}$ if the following conditions hold.

- $\{1, \ldots, N\}$ is the root of $\mathcal{T}$.

- All $\sigma \in V \backslash \mathcal{L}(\mathcal{T})$ are the disjoint union of their sons.

The level of $\sigma \in \mathcal{T}$ is its distance to the root, i.e., the number of son relations that are required for traveling from $\{1, \ldots, N\}$ to $\sigma$. We define the set of clusters on level $j$ as

$$
\mathcal{T}^{(j)}:=\{\sigma \in \mathcal{T}: \sigma \text { has level } j\} .
$$

The construction of the cluster tree is based on the support of the clusters. The support $\Upsilon_{\sigma}$ of a cluster $\sigma$ is defined as the union of the supports of the basis functions corresponding to their elements, that is

$$
\Upsilon_{\sigma}=\bigcup_{i \in \sigma} \Upsilon_{i}, \quad \text { where } \Upsilon_{i}:=\operatorname{supp} \varphi_{i} \text { for all } i \in\{1, \ldots, N\} .
$$

For computing complexity bounds, the cluster tree should match the following additional requirements, uniformly as $N \rightarrow \infty$ :

- The cluster tree is a balanced tree in the sense that the maximal level satisfies $J \sim \log _{2} N$.

- The diameter of the support $\Upsilon_{\sigma_{j}}, \sigma_{j} \in \mathcal{T}^{(j)}$, is local with respect to the level $j$, i.e., $\operatorname{diam} \Upsilon_{\sigma_{j}} \sim 2^{-j / d}$. Moreover, the number \# $\sigma_{j}$ of indices contained in a cluster $\sigma_{j} \in \mathcal{T}^{(j)}$ scales approximately like $2^{J-j}$, i.e., $\# \sigma_{j} \sim 2^{J-j}$.

Until further notice, a binary cluster tree $\mathcal{T}$ with the indicated terms should be given for our further considerations. A common algorithm for its construction is based on a hierarchical subdivision of the point set which is associated with the basis functions, cf., e.g., [37, 38. We begin by embedding the point set $\left\{\mathbf{x}_{1}, \ldots, \mathbf{x}_{N}\right\}$ in a top-level bounding-box. This bounding-box is subsequently subdivided into two cuboids of the same size where the corresponding clusters are described by the points in each bounding-box. This process is iterated until a bounding-box encloses less than a predetermined number of points. In accordance with [41, simplifications can be made by mapping an canonical cluster tree on the reference domain to the surface when a parametric surface representation is used for the boundary $\partial D_{0}$. This situation appears especially in isogeometric analysis, see 42 and the references therein.

\subsection{Hierarchical Matrix Approximation}

For the discretization of kernel functions which are asymptotically smooth in at least one of the variables, we introduce a partition of its domain of definition which separates smooth and non-smooth areas of the kernel function.

Definition 6.2. Two clusters $\sigma$ and $\sigma^{\prime}$ are called admissible if

$$
\max \left\{\operatorname{diam}\left(\Upsilon_{\sigma}\right), \operatorname{diam}\left(\Upsilon_{\sigma^{\prime}}\right)\right\} \leq \eta \operatorname{dist}\left(\Upsilon_{\sigma}, \Upsilon_{\sigma^{\prime}}\right)
$$

holds for some fixed $\eta>0$.

We can obtain the set of admissible blocks by means of a recursive algorithm: Starting with the root $\left(\sigma_{0,0}, \sigma_{0,0}\right)$, the bounding-boxes of the current cluster pair are checked for admissibility. If they are admissible, the cluster pair is added to the set $\mathcal{F}$, which corresponds to the correlation kernel's farfield. Otherwise, the admissibility check will be performed on all bounding-boxes of 
the possible pairs of son clusters of the two original clusters. When we arrive at a pair of leaf clusters with inadmissible bounding-boxes, the clusters are added to the set $\mathcal{N}$, which corresponds to the correlation kernel's nearfield. The set $\mathcal{B}=\mathcal{F} \cup \mathcal{N}$ obviously inherits a tree structure from the recursive construction of $\mathcal{F}$ and $\mathcal{N}$ and is called the block cluster tree, see [37, 38.

With the definition of the block cluster tree at hand, we are finally in the position to introduce $\mathcal{H}$-matrices.

Definition 6.3. The set $\mathcal{H}(\mathcal{B}, k)$ of $\mathcal{H}$-matrices of maximal block rank $k$ is defined according to

$$
\mathcal{H}(\mathcal{B}, k):=\left\{\mathbf{X} \in \mathbb{R}^{N \times N}: \operatorname{rank}\left(\left.\mathbf{X}\right|_{\sigma \times \sigma^{\prime}}\right) \leq k \text { for all }\left(\sigma, \sigma^{\prime}\right) \in \mathcal{F}\right\} .
$$

Note that all nearfield blocks $\left.\mathbf{X}\right|_{\sigma \times \sigma^{\prime}},\left(\sigma, \sigma^{\prime}\right) \in \mathcal{N}$, are allowed to be full matrices.

In accordance with [37, 38, the storage cost of an $\mathcal{H}$-matrix $\mathbf{X} \in \mathcal{H}(\mathcal{B}, k)$ is $\mathcal{O}(k N \log N)$. Here, for correlation kernels which are asymptotically smooth in at least one variable, the rank $k$ depends poly-logarithmically on the desired approximation accuracy $\varepsilon$, which in turn usually depends on the degrees of freedom $N$. While the asymptotical smoothness of explicitely given kernel functions can easily be verified, other kernel functions are the topic of the next subsection.

\subsection{Covariance Calculus}

The compressibility of an implicitly given kernel function, such as $\rho^{(\operatorname{Cor}[\delta u])}$ in $(22)$, has been studied in 21] for the case of smooth domains and manifolds. We restate the main theorem for the setting of the present article which employs that the Hilbert-Schmidt operator

$$
\left(\mathcal{C}_{\langle\mathbf{V}, \mathbf{n}\rangle} \psi\right)(\mathbf{x}):=\int_{\partial D_{0}} \operatorname{Cor}[\langle\mathbf{V}, \mathbf{n}\rangle](\mathbf{x}, \mathbf{y}) \psi(\mathbf{y}) \mathrm{d} \sigma_{\mathbf{y}},
$$

related with the correlation kernel $\operatorname{Cor}[\langle\mathbf{V}, \mathbf{n}\rangle]$, is in general a pseudo-differential operator, see, e.g., 43] and the references therein.

Theorem 6.4. Let $\partial D_{0}$ be an analytic manifold and consider the correlation equation

$$
(\Psi \otimes \Psi) \operatorname{Cor}[u]=\operatorname{Cor}[\langle\mathbf{V}, \mathbf{n}\rangle] .
$$

Assume that the correlation kernel $\operatorname{Cor}[\langle\mathbf{V}, \mathbf{n}\rangle]$ gives rise to an operator $\mathcal{C}_{\langle\mathbf{V}, \mathbf{n}\rangle} \in O P S_{c l, s}^{\theta}$, i.e., to a classical pseudo-differential operator with symbol $a_{\langle\mathbf{V}, \mathbf{n}\rangle}(\mathbf{x}, \boldsymbol{\xi})$ of order $\theta$ and of Gevrey class $s \geq 1$ in the sense of [44, Definition 1.1]. Then, if $\Psi \in O P S_{c l, s}^{r}$ is properly supported, the solution correlation kernel Cor $[u]$ is the Schwartz kernel of an operator $\mathcal{C}_{u} \in O P S_{c l, s}^{\theta-2 r}$.

Moreover, the kernel $\mathrm{Cor}[u]$ of the correlation operator $\mathcal{C}_{u}$ is smooth in local coordinates away from the diagonal, and there holds the pointwise estimate

$$
\left|\partial_{\mathbf{x}}^{\boldsymbol{\alpha}} \partial_{\mathbf{y}}^{\boldsymbol{\beta}} \operatorname{Cor}[u](\mathbf{x}, \mathbf{y})\right| \leq c \mathscr{A}^{|\boldsymbol{\alpha}+\boldsymbol{\beta}|}(|\boldsymbol{\alpha}| !)^{s} \boldsymbol{\beta} !\|\mathbf{x}-\mathbf{y}\|_{2}^{-\theta-n-|\boldsymbol{\alpha}|-|\boldsymbol{\beta}|-2}
$$

in local coordinates away form the diagonal for all $\boldsymbol{\alpha}, \boldsymbol{\beta} \in \mathbb{N}_{0}^{n-1}$, with some constants $c$ and $\mathscr{A}$ which depend only on $M, \kappa$, and $a_{\langle\mathbf{V}, \mathbf{n}\rangle}$.

Obviously, for $s=1$, estimate (35) directly implies condition (32) for the asymptotic smoothness of Cor $[u]$, whereas $s>1$ implies the asymptotic smoothness in one variable. We can thus approximate $\operatorname{Cor}[u]$ by means of $\mathcal{H}$-matrices. The assumption of an analytic manifold can be relaxed to a manifold of Gevrey class $s$ in the case $s>1$. In particular, 21 provides also some numerical evidence that the result could likely be extended to Lipschitz domains. An example of correlation kernels for $\operatorname{Cor}_{\langle\mathbf{V}, \mathbf{n}\rangle}$, which satisfy the conditions of this theorem for $s \geq 1$, is again the Matérn class of kernels. We refer to [21] for more details on how to verify the assumptions of the theorem for other kernel functions.

Theorem 6.4 does not immediately show that the kernel functions $A$ from $\sqrt{19})$ and $\rho^{(\operatorname{Cor}[\delta u])}$ from (22) are asymptotically smooth, but it can be extended to the following slightly modified version. 
Corollary 6.5. Let $\partial D_{0}$ be a manifold of Gevrey class $s>1$ or an analytic manifold (i.e. $s=1$ ), and let $\operatorname{Cor}[\langle\mathbf{V}, \mathbf{n}\rangle]$ fulfil the same requirements as in Theorem 6.4 for $s \geq 1$. Let further $g$ be analytic in the case $s=1$ and of Gevrey class $s>1$ otherwise. Then, the solutions of the equations (19) and 22) are asymptotically smooth functions for $s=1$ and asymptotically smooth in one variable for $s>1$.

Proof. We first remark that the single layer operator belongs to $O P S_{c l, s}^{-1}$ and the double layer operator belongs to $O P S_{c l, s}^{0}$ for $s \geq 1$, cf. [43]. Let us further remark that the multiplication of two functions $g(\mathbf{x}) f(\mathbf{x})$ can be written as an application of an integral operator

$$
g(\mathbf{x}) f(\mathbf{x})=\int_{\partial D_{0}} k(\mathbf{x}, \mathbf{x}-\mathbf{y}) f(\mathbf{y}) \mathrm{d} \sigma_{\mathbf{y}}=(\Psi f)(\mathbf{x}),
$$

with distributional Schwartz kernel $k(\mathbf{x}, \mathbf{x}-\mathbf{y})=g(\mathbf{x}) \delta(\mathbf{x}-\mathbf{y})$, with $\delta$ denoting the delta distribution. Since the Fourier transform of the delta distribution is a constant, the multiplication by a function gives thus rise to a pseudo-differential operator in $O P S_{c l, s}^{0}$. Rewriting finally $(19)$ as

$$
(\operatorname{Id} \otimes \mathcal{V}) A=\left(\operatorname{Id} \otimes\left(\frac{1}{2}+\mathcal{K}\right)\right)\left(\left(\mathbb{1} \otimes\left(\frac{\partial g}{\partial \mathbf{n}}-t^{\left(u_{0}\right)}\right) \operatorname{Cor}[\langle\mathbf{V}, \mathbf{n}\rangle]\right),\right.
$$

the rest of the proof is in complete analogy to [21, Theorems 1,2, and 3].

Since sparse matrices corresponding to local operators can easily be represented by $\mathcal{H}$-matrices, it is thus reasonable to compress all matrices in $(27)$ and $(28)$ as $\mathcal{H}$-matrices. However, it remains to explain how to actually compute the representations for $\mathbf{C}_{\rho, \operatorname{Cor}[\delta u]}$ in $(27)$ and $\mathbf{A}$ in $(28)$.

\section{5. $\mathcal{H}$-Matrix Arithmetic and Iterative Solution}

An important feature of $\mathcal{H}$-matrices is that efficient algorithms for approximate matrix arithmetic operations are available. For two $\mathcal{H}$-matrices $\mathbf{H}_{1}, \mathbf{H}_{2} \in \mathcal{H}(\mathcal{B}, k)$, the approximate matrixmatrix addition $\mathbf{H}_{1}+\mathbf{H}_{2} \in \mathcal{H}(\mathcal{B}, k)$ can be performed in $\mathcal{O}\left(k^{2} N \log N\right)$ operations, while the approximate matrix-matrix multiplication $\mathbf{H}_{1} * \mathbf{H}_{2} \in \mathcal{H}(\mathcal{B}, k)$ can be performed in $\mathcal{O}\left(k^{2} N \log ^{2} N\right)$ operations. Both of these operations are essentially block matrix algorithms with successive recompression schemes. Moreover, employing the recursive block structure, the approximate inversion or the approximate computation of the $L U$-decomposition within $\mathcal{H}(\mathcal{B}, k)$ can also be performed in only $\mathcal{O}\left(k^{2} N \log ^{2} N\right)$ operations. We refer the reader to [23, 37, 38, 45] for further results and implementation details.

In the context of correlation equations, this approximate $\mathcal{H}$-matrix arithmetic has successfully been used in [19, 20, to solve correlation equations as (34), where the operator $\Psi$ has been a differential operator in a domain or a boundary integral operator on a domain's boundary. The resulting matrix equation has been solved by using an iterative solver based on iterative refinement, cf. 46, 47, 48, which we are also going to employ here and has originally been introduced in 48] for the improvement of solutions to linear systems of equations based on the LU-factorization.

More general, having all matrices in the general matrix equation $\mathbf{\Psi} \mathbf{X} \boldsymbol{\Phi}^{\top}=\mathbf{R}$ represented by $\mathcal{H}$-matrices, the solution is derived as follows. Let $\boldsymbol{\Xi} \approx \hat{\mathbf{L}}_{\boldsymbol{\Xi}} \hat{\mathbf{U}}_{\boldsymbol{\Xi}}$, where $\hat{\mathbf{L}}_{\boldsymbol{\Xi}}, \hat{\mathbf{U}}_{\boldsymbol{\Xi}} \in \mathcal{H}(\mathcal{B}, k)$, $\boldsymbol{\Xi} \in\{\boldsymbol{\Psi}, \boldsymbol{\Phi}\}$, be approximate LU-decompositions to $\boldsymbol{\Psi}$ and $\boldsymbol{\Phi}$, e.g., computed by the $\mathcal{H}$-matrix arithmetic. Starting with the initial guess $\mathbf{X}_{0}=\hat{\mathbf{U}}_{\boldsymbol{\Psi}}^{-1} \hat{\mathbf{L}}_{\boldsymbol{\Psi}}^{-1} \mathbf{R} \hat{\mathbf{L}}_{\boldsymbol{\Phi}}^{-\boldsymbol{\top}} \hat{\mathbf{U}}_{\boldsymbol{\Phi}}^{-\boldsymbol{\top}}$, we iterate

$$
\boldsymbol{\Theta}_{i}=\mathbf{R}-\boldsymbol{\Psi} \mathbf{X}_{i} \boldsymbol{\Phi}^{\top}, \quad \mathbf{X}_{i+1}=\mathbf{X}_{i}+\hat{\mathbf{U}}_{\boldsymbol{\Psi}}^{-1} \hat{\mathbf{L}}_{\boldsymbol{\Psi}}^{-1} \boldsymbol{\Theta}_{i} \hat{\mathbf{L}}_{\boldsymbol{\Phi}}^{-\top} \hat{\mathbf{U}}_{\boldsymbol{\Phi}}^{-\boldsymbol{\top}}, \quad i=0,1, \ldots
$$

The idea of iterative refinement is that the residual $\boldsymbol{\Theta}_{i}$ is computed with a higher precision than the correction $\hat{\mathbf{U}}_{\boldsymbol{\Psi}}^{-1} \hat{\mathbf{L}}_{\boldsymbol{\Psi}}^{-1} \boldsymbol{\Theta}_{i} \hat{\mathbf{L}}_{\boldsymbol{\Phi}}^{-\boldsymbol{\top}} \hat{\mathbf{U}}_{\boldsymbol{\Phi}}^{-\boldsymbol{\top}}$. This yields an improvement of the iterate in each step. Note that this algorithm also algebraically coincides with an undamped preconditioned Richardson iteration.

Before we conclude this section, we want to remark that the approximate LU-decomposition can be replaced by an approximate Cholesky decomposition if the corresponding matrix is symmetric and positive definite, such as the system matrix of the single layer operator. 

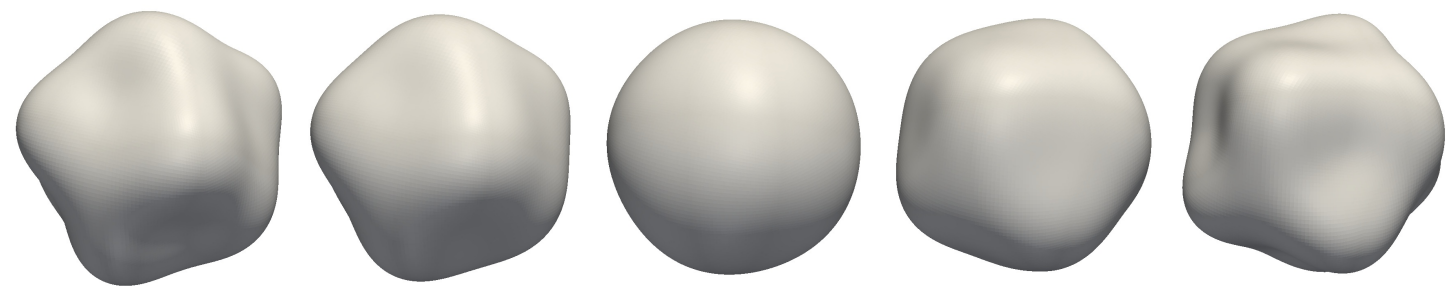

Figure 2: Different realizations of the jiggled unit sphere for the spherical harmonic $Y_{3}^{5}$ and $\varepsilon=0.3$.

\section{Numerical Examples}

The following numerical experiments are divided into three parts. The first part is concerned with the convergence of the Galerkin scheme for the correction terms $\operatorname{Cor}[\delta u]_{h}$ and $\mathbb{E}\left[\delta^{2} u\right]_{h}$ with respect to the mesh size $h$. The second part is concerned with the asymptotics of the perturbation approach in $\varepsilon$, whereas the third part deals with an example on non-smooth domains.

All of the computations in the following experiments were carried out on a computing server with two Intel(R) Xeon(R) CPU E5-2643 v3 with a clock rate of $3.40 \mathrm{GHz}$ and a main memory of 256GB. Each of the CPUs provides 12 physical cores, thus, with Hyper-Threading enabled, we may access up to 24 cores in total. For the discretization and the assembly of the $\mathcal{H}$-matrices, we employ the black-box higher order fast $\mathcal{H}^{2}$-multipole method proposed in [41. The computations in the tensor product domain $\partial D_{0} \times \partial D_{0}$ are based on discontinuous (elementwise) polynomial ansatz functions and the computations in the non-product domain on globally continuous B-splines of the same order. For the arithmetic $\mathcal{H}$-matrix operations, we apply the $\mathcal{H}$-matrix arithmetic tailored to parametric surfaces as described in [19, where the almost linear scaling with respect to $N$ has been verified. We employ a simple OpenMP parallelization to accelerate the computations and refer to [49, where a more sophisticated parallelization has been discussed.

\subsection{Convergence in $h$}

To construct an example, where the solution is analytically known, we consider the unit ball whose boundary is perturbed in normal direction in accordance with

$$
\varepsilon\left\langle\mathbf{V}(\omega, \mathbf{x}), \mathbf{n}_{\mathbf{x}}\right\rangle=Y_{m}^{\ell}(\mathbf{x}) X(\omega),
$$

where $Y_{m}^{\ell}$ is a spherical harmonic and $X(\omega)$ is a uniformly distributed random variable on $[-\varepsilon, \varepsilon]$. The correlation of this boundary perturbation thus implies

$$
\operatorname{Cor}[\langle\mathbf{V}, \mathbf{n}\rangle](\mathbf{x}, \mathbf{y})=\frac{1}{3} Y_{m}^{\ell}(\mathbf{x}) Y_{m}^{\ell}(\mathbf{y}), \quad \mathbf{x}, \mathbf{y} \in \partial D_{0} .
$$

Several possible shapes are depicted in Figure 2, while the used compactum $K$ was visualized in Figure 1 .

As boundary values on the hold-all domain, we choose

$$
g(\mathbf{x})=\|\mathbf{x}\|^{2}, \quad \mathbf{x} \in D_{\varepsilon}^{\cup \Omega} .
$$

Since $\|\mathbf{x}\|^{\ell} Y_{m}^{\ell}(\mathbf{x} /\|\mathbf{x}\|)$ is harmonic in $\mathbb{R}^{3}$, it is then a short computation to show that it holds

$$
\operatorname{Cor}[\delta u](\mathbf{x}, \mathbf{y})=\frac{4}{3}\|\mathbf{x}\|^{\ell}\|\mathbf{y}\|^{\ell} Y_{m}^{\ell}\left(\frac{\mathbf{x}}{\|\mathbf{x}\|}\right) Y_{m}^{\ell}\left(\frac{\mathbf{y}}{\|\mathbf{y}\|}\right), \quad \mathbf{x}, \mathbf{y} \in D_{0} .
$$

Since the spherical harmonic $Y_{m}^{\ell}$ is an eigenfunction of the Dirichlet-to-Neumann map with eigenvalue $\ell$ on the sphere, cf. [50], and since the unit sphere has the constant mean curvature of $\mathcal{H}=-1$, cf. [51, one verifies that there holds

$$
g^{\left(\mathbb{E}\left[\delta^{2} u\right]\right)}=\frac{(2-4 \ell)}{3}\left(Y_{m}^{\ell}\right)^{2} \quad \text { on } \partial D_{0},
$$



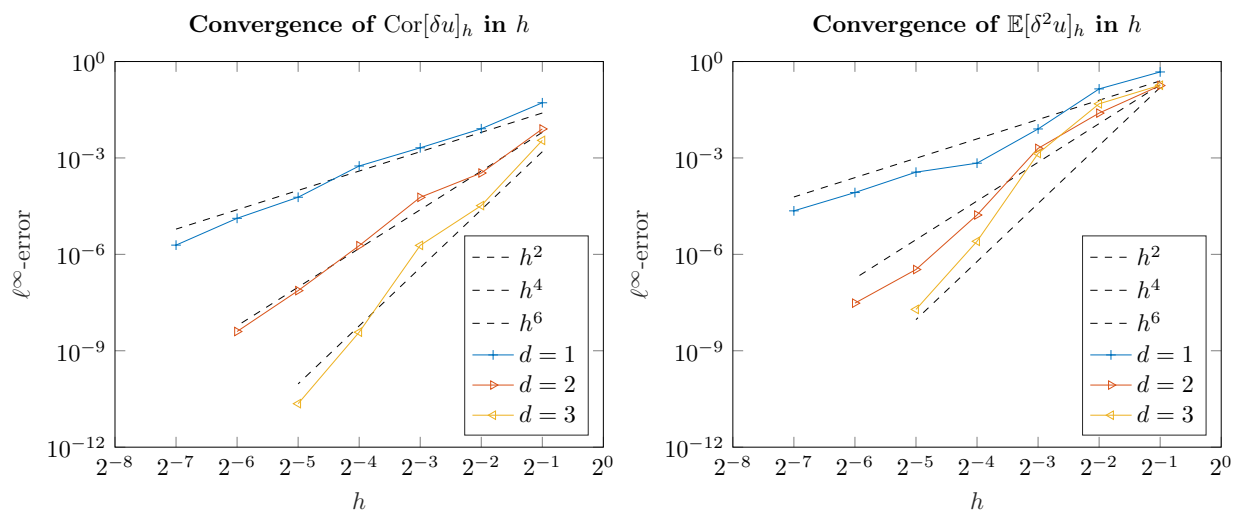

Figure 3: Convergence rates for $\operatorname{Cor}[\delta u]$ and $\mathbb{E}\left[\delta^{2} u\right]$ on the jiggled unit ball for different orders $d$ of the ansatz functions.

for the Dirichlet data of the mean's second order correction term $\mathbb{E}\left[\delta^{2} u\right]$ from $(13)$. Having thus access to the exact Dirichlet data, we can easily compute an overkill solution of $\mathbb{E}\left[\overline{\delta^{2}} u\right]$ as a reference solution by an additional refinement in $h$.

Figure 3 validates that we achieve the convergence rates predicted by Lemma 5.6 and Lemma 5.11 for both, $\operatorname{Cor}[\delta u]$ and $\mathbb{E}\left[\delta^{2} u\right]$. In fact, $\mathbb{E}\left[\delta^{2} u\right]$ has even a higher convergence rate than predicted. Although not covered by our theory, the case $d=1$, i.e., the case of piecewise constant boundary elements, seems to converge as well at a rate of $h^{2}$. Notice that the computed quantities are independent of the amplitude of the jiggling $\varepsilon$, as long as the compactum $K \Subset D_{\varepsilon}^{\cap \Omega} \operatorname{does}$ not depend on $\varepsilon$.

\subsection{Asymptotics in $\varepsilon$}

As the mean, the correlation, and the covariance for the example on the unit ball are not analytically known, we have to use quadrature methods to compute a reference solution in order to study the asymptotics in $\varepsilon$. Choosing $\varepsilon=0.05,0.1,0.15,0.2,0.25,0.3$, we use a one-dimensional 15 point Gauss-Legendre quadrature rule to compute the stochastic integral of the mean, the correlation, and the covariance on a spatial discretization with continuous piecewise linear ansatz functions on a mesh with 24,576 elements. The PDE solves are accelerated by an OpenMP parallelization.

Since the probability distribution of $\langle\mathbf{V}, \mathbf{n}\rangle$ is symmetric around zero, we can expect fourth order accurate approximations to the mean, the correlation, and the covariance in $\varepsilon$. Thus, for the numerical solutions $\mathbb{E}\left[u_{\varepsilon}\right]_{h}$, Cor $\left[u_{\varepsilon}\right]_{h}$, and $\operatorname{Cov}\left[u_{\varepsilon}\right]_{h}$ computed by our expansions, the errors should behave like

$$
\begin{aligned}
\left|\mathbb{E}\left[u_{\varepsilon}\right]-\mathbb{E}\left[u_{\varepsilon}\right]_{h}\right| & =\mathcal{O}\left(h^{2 d}+\varepsilon^{2} h^{2(d-1)}+\varepsilon^{4}\right) & & \text { in } K, \\
\left|\operatorname{Cor}\left[u_{\varepsilon}\right]-\operatorname{Cor}\left[u_{\varepsilon}\right]_{h}\right| & =\mathcal{O}\left(h^{2 d}+\varepsilon^{2} h^{2 d}+\varepsilon^{4}\right) & & \text { in } K \times K, \\
\left|\operatorname{Cov}\left[u_{\varepsilon}\right]-\operatorname{Cov}\left[u_{\varepsilon}\right]_{h}\right| & =\mathcal{O}\left(\varepsilon^{2} h^{2 d}+\varepsilon^{4}\right) & & \text { in } K \times K,
\end{aligned}
$$

as $h$ and $\varepsilon$ tend to zero. The fourth order asymptotic is then reached as soon as the mesh size is small enough.

Figure 4 shows the errors for the former second order (see [7] for the details) and the new fourth order accurate approximations. We indeed reach the fourth order accuracy already for relatively coarse mesh sizes. A comparison with the second order accurate approximation shows that the consideration of the correction terms for the fourth order approximation can improve the error by several orders of magnitude.

\subsection{Non-smooth Boundaries}

In order to demonstrate that the perturbation approach is not necessarily limited to smooth surfaces, we consider the unit cube $D_{0}=[0,1]^{3}$ as the reference domain. We assume the perturbing 
second order accurate $\varepsilon$-asymptotics of $\mathbb{E}\left[u_{\varepsilon}\right]_{h}$

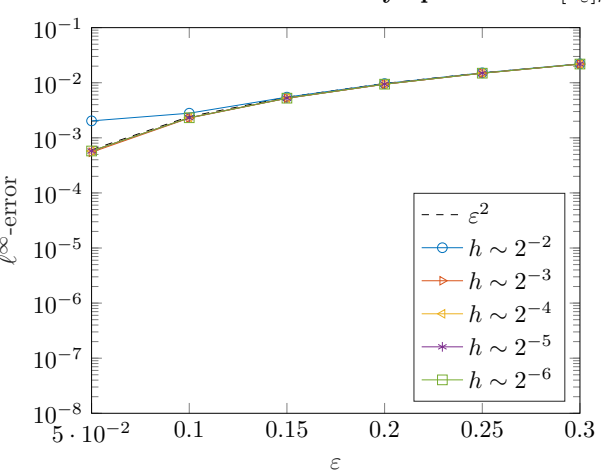

fourth order accurate $\varepsilon$-asymptotics of $\mathbb{E}\left[u_{\varepsilon}\right]_{h}$

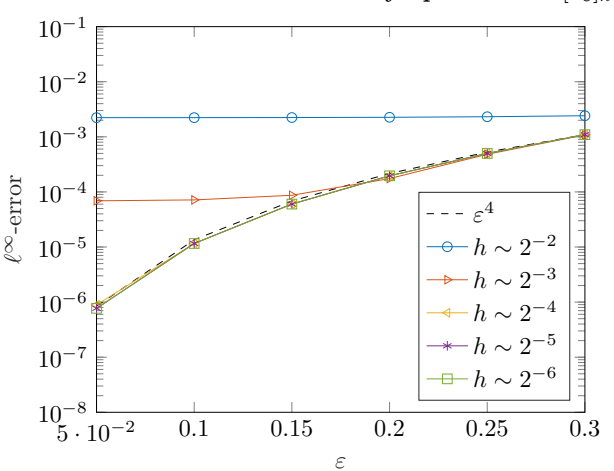

second order accurate $\varepsilon$-asymptotics of $\operatorname{Cor}\left[u_{\varepsilon}\right]_{h}$

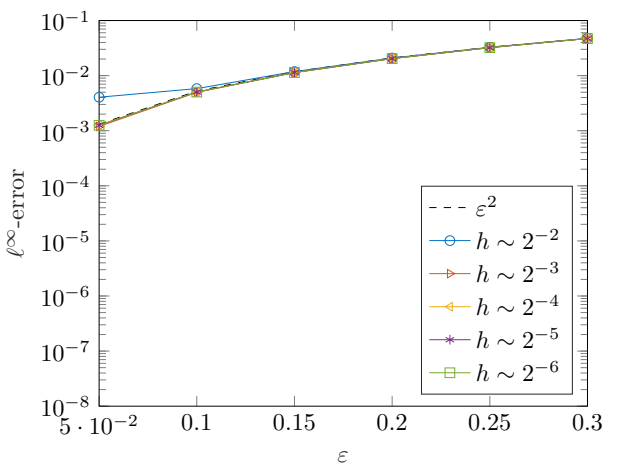

fourth order accurate $\varepsilon$-asymptotics of $\operatorname{Cor}\left[u_{\varepsilon}\right]_{h}$

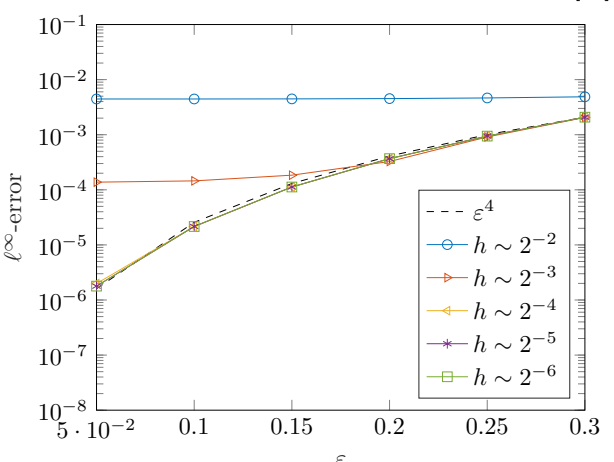

second order accurate $\varepsilon$-asymptotics of $\operatorname{Cov}\left[u_{\varepsilon}\right]_{h}$

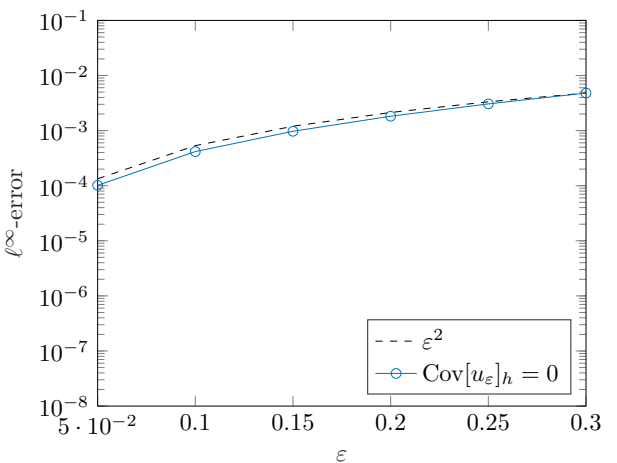

fourth order accurate $\varepsilon$-asymptotics of $\operatorname{Cov}\left[u_{\varepsilon}\right]_{h}$

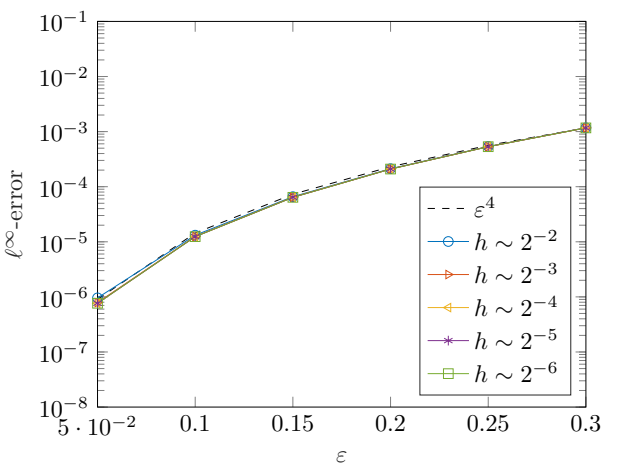

Figure 4: Asymptotics in $\varepsilon$ for the numerical approximation of the second order and fourth order accurate expansions for the mean, the correlation, and the covariance by different mesh sizes $h$. 

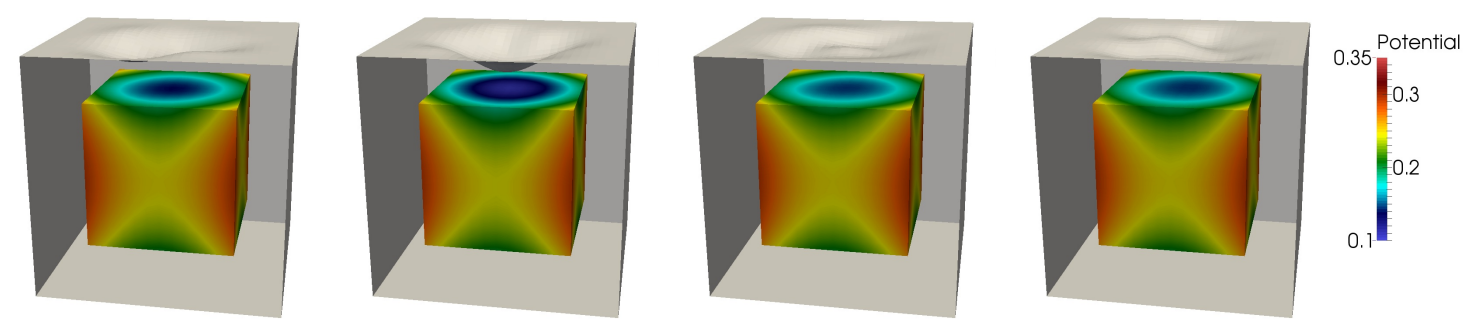

Figure 5: Realizations of the perturbed cube with its solution visualized on the inscribed compactum $K$ for $\varepsilon=0.05$.

vector field to jiggle the upper side of the cube. More precisely, given uniformly distributed random variables $X_{i j} \sim U[-1,1], i, j=1, \ldots, 4$, the perturbation field is given as

$$
\mathbf{V}(\omega, \mathbf{x})= \begin{cases}\sum_{i, j=1}^{4} B_{i}\left(x_{1}\right) B_{j}\left(x_{2}\right) X_{i j}(\omega), & x_{3}=1 \\ 0, & \text { otherwise }\end{cases}
$$

where $B_{i}, i=1, \ldots, 4$ are fifth order B-splines on $[0,1]$, which, as well as their derivatives, are zero at the interval boundaries. As a result, the shape derivatives are well defined and no singularities occur in the boundary values of the PDEs for the correction terms, which means that the expansions for the mean, the correlation, and the covariance are well defined.

A visualization of different realizations for $\varepsilon=0.05$ for the Dirichlet boundary values

$$
g(\mathbf{x})=\left(x_{1}-0.5\right)^{2}+\left(x_{2}-0.5\right)^{2}
$$

and the used compactum $K$ with the corresponding solution of $(3)$ is found in Figure 5 .

Since the contributions of all 16 boundary perturbations are equally important, the dimension for the stochastic integrals for our quantities of interest is truly 16-dimensional. Therefore, we use a Monte-Carlo simulation with $M=10,000$ samples for a visual comparison with the asymptotic expansions for the choice $\varepsilon=0.05$. Then, the error of a Monte-Carlo approximation $X_{M}$ to $X$, computed from $M$ samples, is given by

$$
\mathbb{E}\left[\left|X-X_{M}\right|\right] \leq \sqrt{\frac{\mathbb{V}[X]}{M}} .
$$

All computations are performed by using continuous piecewise linear ansatz functions on a mesh with 24,576 elements, while we accelerate the Monte-Carlo simulation by computing only the changed matrix entries for each sample. The computation time on all 24 cores takes for all quantities 67'241 seconds using the perturbation approach and 496'582 seconds using the MonteCarlo simulation. Still, due to the slow convergence rate of the Monte-Carlo simulation and the high-dimensionality of the problem, we only aim at a qualitative comparison of the mean and the diagonal of the correlation, which is presented in Figure 6.

\section{Conclusion}

In this article, we considered the approximate computation of the mean, the correlation, and the covariance of the solution of PDEs on random domains by the perturbation approach. Additionally to existing third order accurate expansions of the covariance discussed in [7, we derived third order accurate expansions of the mean and the correlation and discussed their numerical computation. These expansions become even fourth order accurate for specific types of boundary variations. While the solution on the unperturbed domain yields a second order accurate solution, the correction terms for the more accurate expansions are given by correlation equations.

Omitting the meshing of the computational domain, we employ the boundary element method to discretize the correlation equations with ansatz spaces of piecewise polynomials of at least 

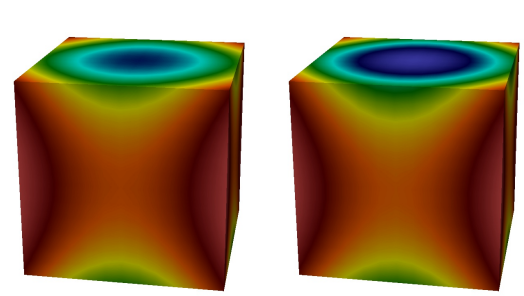

Mean
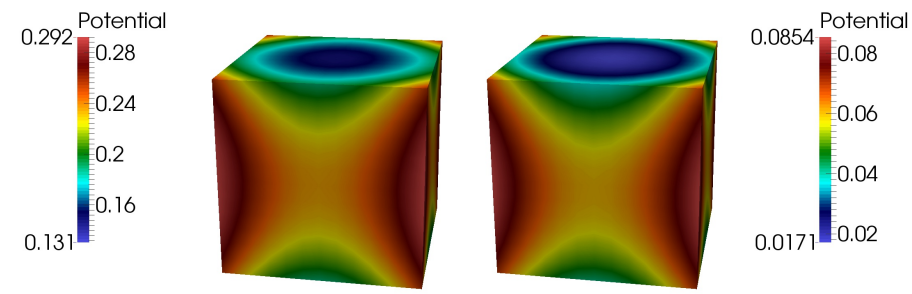

Correlation

Figure 6: Comparison between the perturbation approach (left) and a Monte-Carlo simulation (right) with 10'000 samples for $\varepsilon=0.05$.

second order. To obtain computational efficiency, we solve the corresponding matrix equations in almost linear complexity by the $\mathcal{H}$-matrix approach. The numerical experiments in three dimensions validate the asymptotic expansions and show the convergence of the proposed method for piecewise linear and piecewise quadratic boundary elements. Piecewise constant boundary elements, although not covered by the theory, seem to work as well. Finally, we provided numerical experiments which indicate how the perturbation approach could be applied on nonsmooth domains.

\section{References}

[1] J. E. Castrillón-Candás, F. Nobile, R. F. Tempone, Analytic regularity and collocation approximation for elliptic PDEs with random domain deformations, Computers \& Mathematics with Applications 71 (6) (2016) 1173-1197.

[2] H. Harbrecht, M. Peters, M. Siebenmorgen, Analysis of the domain mapping method for elliptic diffusion problems on random domains, Numerische Mathematik 134 (4) (2016) 823-856.

[3] D. Xiu, D. M. Tartakovsky, Numerical methods for differential equations in random domains, SIAM Journal on Scientific Computing 28 (3) (2006) 1167-1185.

[4] A. Chernov, D. Pham, T. Tran, A shape calculus based method for a transmission problem with a random interface, Computers \& Mathematics with Applications 70 (7) (2015) 1401-1424.

[5] H. Harbrecht, J. Li, First order second moment analysis for stochastic interface problems based on low-rank approximation, ESAIM: Mathematical Modelling and Numerical Analysis 47 (5) (2013) 1533-1552.

[6] H. Harbrecht, M. Peters, The second order perturbation approach for PDEs on random domains, Applied Numerical Mathematics 125 (2018) 159-171.

[7] H. Harbrecht, R. Schneider, C. Schwab, Sparse second moment analysis for elliptic problems in stochastic domains, Numerische Mathematik 109 (3) (2008) 385-414.

[8] J. E. Castrillón-Candás, F. Nobile, R. F. Tempone, Hybrid collocation perturbation for PDEs with random domains, Preprint arXiv:1703.10040.

[9] M. C. Delfour, J. P. Zolésio, Shapes and Geometries: Metrics, Analysis, Differential Calculus, and Optimization, 2nd Edition, Vol. 22 of Advances in Design and Control, SIAM, Philadelphia, PA, 2011.

[10] J. Sokolowski, J. P. Zolésio, Introduction to Shape Optimization. Shape Sensitivity Analysis, Vol. 16 of Springer series in computational mathematics, Springer, Berlin-Heidelberg, 1992.

[11] H. Harbrecht, A finite element method for elliptic problems with stochastic input data, Applied Numerical Mathematics 60 (3) (2010) 227-244.

[12] H. Harbrecht, M. Peters, R. Schneider, On the low-rank approximation by the pivoted Cholesky decomposition, Applied Numerical Mathematics 62 (2012) 28-440.

[13] H. Harbrecht, R. Schneider, C. Schwab, Multilevel frames for sparse tensor product spaces, Numerische Mathematik 110 (2) (2008) 199-220.

[14] C. Schwab, R. A. Todor, Sparse finite elements for elliptic problems with stochastic loading, Numerische Mathematik 95 (4) (2003) 707-734.

[15] C. Schwab, R. A. Todor, Sparse finite elements for elliptic problems with stochastic loading, Numerische Mathematik 95 (4) (2003) 707-734.

[16] T. von Petersdorff, C. Schwab, Sparse finite element methods for operator equations with stochastic data, Applications of Mathematics 51 (2) (2006) 145-180.

[17] M. Griebel, H. Harbrecht, Approximation of bi-variate functions: singular value decomposition versus sparse grids, IMA Journal of Numerical Analysis 34 (1) (2014) 28-54.

[18] C. Schwab, R. A. Todor, Karhunen-Loève approximation of random fields by generalized fast multipole methods, Journal of Computational Physics 217 (2006) 100-122.

[19] J. Dölz, H. Harbrecht, M. Peters, $\mathcal{H}$-matrix accelerated second moment analysis for potentials with rough correlation, Journal of Scientific Computing 65 (1) (2015) 387-410. 
[20] J. Dölz, H. Harbrecht, M. Peters, $\mathcal{H}$-matrix based second moment analysis for rough random fields and finite element discretizations, SIAM Journal on Scientific Computing 39 (4) (2017) B618-B639.

[21] J. Dölz, H. Harbrecht, C. Schwab, Covariance regularity and $\mathcal{H}$-matrix approximation for rough random fields, Numerische Mathematik 135 (4) (2017) 1045-1071.

[22] W. Hackbusch, A sparse matrix arithmetic based on $\mathcal{H}$-matrices part I: Introduction to $\mathcal{H}$-matrices, Computing 62 (2) (1999) 89-108.

[23] W. Hackbusch, B. N. Khoromskij, A sparse $\mathcal{H}$-matrix arithmetic. General complexity estimates, Journal of Computational and Applied Mathematics 125 (1-2) (2000) 479-501.

[24] J. Wloka, Partial Differential Equations, Cambridge University Press, Cambridge, 1987.

[25] K. Eppler, Boundary integral representations of second derivatives in shape optimization, Discussiones Mathematicae, Differential Inclusions, Control and Optimization 20 (1) (2000) 63-78.

[26] M. Dambrine, On variations of the shape Hessian and sufficient conditions for the stability of critical shapes, RACSAM, Revista de la Real Academia de Ciencias Exactas, Físicas y Naturales. Serie A. Matemáticas 96 (1) (2002) 95-121.

[27] M. Loève, Probability Theory, 4th Edition, Vol. II, Springer, New York, 1978.

[28] O. Steinbach, Numerical Approximation Methods for Elliptic Boundary Value Problems, Springer Science + Business, New York, 2008

[29] A. Behzadan, M. Holst, Multiplication in Sobolev spaces, revisited, Preprint arXiv:1512.07379.

[30] J. P. Zolésio, Multiplication dans les espaces de Besov, Proceedings of the Royal Society of Edinburgh: Section A Mathematics 78 (1-2) (1977) 113-117.

[31] M. Dambrine, C. Dapogny, H. Harbrecht, Shape optimization for quadratic functionals and states with random right-hand sides, SIAM Journal on Control and Optimization 53 (5) (2015) 3081-3103.

[32] L. Greengard, V. Rokhlin, A fast algorithm for particle simulations, Journal of Computational Physics 73 (2) (1987) 325-348

[33] W. Hackbusch, Z. P. Nowak, On the fast matrix multiplication in the boundary element method by panel clustering, Numerische Mathematik 54 (4) (1989) 463-491.

[34] G. Beylkin, R. Coifman, V. Rokhlin, Fast wavelet transforms and numerical algorithms I, Communications on Pure and Applied Mathematics 44 (2) (1991) 141-183.

[35] W. Dahmen, H. Harbrecht, R. Schneider, Compression techniques for boundary integral equations. Asymptotically optimal complexity estimates, SIAM Journal on Numerical Analysis 43 (6) (2006) 2251-2271.

[36] M. Bebendorf, Approximation of boundary element matrices, Numerische Mathematik 86 (4) (2000) 565-589.

[37] S. Börm, Efficient Numerical Methods for Non-local Operators, Vol. 14 of EMS Tracts in Mathematics, European Mathematical Society (EMS), Zürich, 2010

[38] W. Hackbusch, Hierarchical Matrices: Algorithms and Analysis, Springer, Heidelberg, 2015.

[39] B. Matérn, Spatial Variation, Lecture Notes in Statistics, Springer, New York, 1986.

[40] C. E. Rasmussen, C. K. I. Williams, Gaussian Processes for Machine Learning (Adaptive Computation and Machine Learning), The MIT Press, Cambridge, 2005.

[41] J. Dölz, H. Harbrecht, M. Peters, An interpolation-based fast multipole method for higher-order boundary elements on parametric surfaces, International Journal for Numerical Methods in Engineering 108 (13) (2016) 1705-1728.

[42] J. A. Cottrell, T. J. R. Hughes, Y. Bazilevs, Isogeometric Analysis: Toward Integration of CAD and FEA, 1st Edition, Wiley Publishing, 2009

[43] G. C. Hsiao, W. L. Wendland, Boundary Integral Equations, Vol. 164 of Applied Mathematical Sciences, Springer, Berlin, 2008

[44] L. Boutet de Monvel, P. Krée, Pseudo-differential operators and Gevrey classes, Annales de l'Institut Fourier (Grenoble) 17 (fasc. 1) (1967) 295-323.

[45] L. Grasedyck, W. Hackbusch, Construction and arithmetics of $\mathcal{H}$-matrices, Computing 70 (4) (2003) 295-334.

[46] G. H. Golub, C. F. Van Loan, Matrix Computations, 4th Edition, Johns Hopkins University Press, Baltimore, 2012

[47] C. B. Moler, Iterative refinement in floating point, Journal of the ACM 14 (2) (1967) 316-321.

[48] J. H. Wilkinson, Rounding Errors in Algebraic Processes, Prentice Hall, Englewood Cliffs, NJ, 1963.

[49] R. Kriemann, Parallel $\mathcal{H}$-matrix arithmetics on shared memory systems, Computing 74 (3) (2005) $273-297$.

[50] D. Colton, R. Kress, Inverse Acoustic and Electromagnetic Scattering Theory, Vol. 93 of Applied Mathematical Sciences, Springer Science \& Business Media, 2012

[51] A. Gray, Modern Differential Geometry of Curves and Surfaces with Mathematica, CRC Press, Boca Raton, Fl, 2006. 